\title{
Comparative Evaluation of Statistical and Fractal Approaches for JRC Calculation Based on a Large Dataset of Natural Rock Traces
}

\author{
Kristofer Marsch ${ }^{1}$ (D) . Tomas M. Fernandez-Steeger ${ }^{1}$ (D)
}

Received: 1 February 2020 / Accepted: 15 December 2020 / Published online: 3 February 2021

(c) The Author(s) 2021

\begin{abstract}
After the publication of the type-profiles for the estimation of the joint roughness coefficient (JRC) a discussion evolved about how to adequately use these traces. Based on the chart numerous researchers assembled mathematical correlations with various parameters seeking objectivity in the determination of JRC. Within these works differences concerning the database and the mathematical implementations exist. Consequently, each correlation, although predominantly the same parameters are used, leads to different JRC values. In theory, for any arbitrary profile, irrespective of the particular calculation approach, the same JRC should result. This is a requisite because of the referencing of all correlations to the 10 type-profiles. However, it is shown in this study that in most cases equal or even satisfactorily similar results are not obtained. The discrepancies are vast when non-standard profiles are evaluated, in this case, more than 40,000 traces from six different rock surfaces that cover a broad range of roughness categories. The simple intuitive parameter $Z_{2}$ served as an agent for the statistical methods because of its broad use and consequently good comparability. On the part of the fractal approaches, three definitions were used. However, JRC inferred from fractal correlations are very much dependent on the particular calculation routine. In fact, the theory of fractals is overly complex for the sparse and low-resolution type-profiles. In summary, fractal approaches do not produce safer or more reliable estimates of roughness compared to simple statistical means and using $Z_{2}$ perfectly suffices to determine the class of JRC.
\end{abstract}

Keywords Compass walking $\cdot \mathrm{FFT} \cdot$ Spectral analysis $\cdot$ RMS correlation $\cdot Z_{2} \cdot$ Shear strength

$\begin{array}{ll}\text { Abbreviations } \\ \text { JRC } & \begin{array}{l}\text { Joint roughness coefficient (possible sub- } \\ \text { scripts refer to calculation method) }\end{array} \\ D & \begin{array}{l}\text { Fractal dimension (possible subscripts refer } \\ \text { to calculation method) }\end{array} \\ H & \begin{array}{l}\text { Hurst exponent (possible subscripts refer to } \\ \text { calculation method) }\end{array} \\ \text { FFT } & \begin{array}{l}\text { Fast Fourier transform } \\ \text { RMS }\end{array} \\ \text { RMS-COR } & \begin{array}{l}\text { Root-mean-square correlation function/ } \\ \text { method }\end{array} \\ \sigma \delta h(\mathrm{~d} x) & \begin{array}{l}\text { Asperity measure (magnitude parameter) } \\ \text { Asperity measure for 1 mm sampling } \\ \sigma \delta h(1 \mathrm{~mm})\end{array} \\ Z_{2} & \begin{array}{l}\text { lengths } \\ \text { Textural slope parameter (RMS of the aver- } \\ \text { age local slope) }\end{array}\end{array}$

Kristofer Marsch

kristofer.marsch@tu-berlin.de

1 Engineering Geology, Technische Universität Berlin, Berlin, Germany

$\begin{array}{ll}r & \text { Compass radius } \\ R_{\mathrm{p}} & \text { Roughness profile index } \\ \mathrm{SF} & \text { Structure function } \\ \sigma_{\mathrm{i}} & \text { Standard deviation of the slope angles of } \\ & \text { each sampling step } \\ \lambda & \text { Ultimate slope of the profile }\end{array}$

\section{Introduction}

Since the introduction of Barton's empirical shear strength criterion in 1973 there has been a discussion about how to reliably determine the joint roughness coefficient (JRC) as a mechanically meaningful measure for rock surface roughness. Barton and Choubey (1977) published a chart containing 10 type-profiles to estimate the JRC by visual comparison. In doing so, the JRC, being a parameter rather of mechanical nature that shall embrace all effects of roughness within shearing processes, is determined from morphological surface roughness along a profile, of course preferably in the shear direction. The initial intention was to enable 
engineers and geologists to "make a quick estimate" (Barton and Choubey 1977) of JRC without conducting sophisticated mechanical direct shear tests.

However, this practice of roughness determination was questioned since the human eye-based visual comparison is error-prone and highly subjective (e.g. Beer et al. 2002; Alameda-Hernandez et al. 2014). Therefore, it seems reasonable to eliminate the user's influence by applying mathematical means. In the case of surface roughness, this is a common approach in engineering and material sciences (e.g. ISO 1997). Consequently, based on the standard chart numerous researchers assembled mathematical correlations with various parameters seeking objectivity in the determination of JRC. Tse and Cruden (1979) were the first to calculate statistical parameters for the type-profiles and to correlate them with JRC. Since then, many authors have published a still growing number of correlations of other statistical parameters. Li and Zhang (2015) give a rather recent overview of these endeavours. Apart from statistical approaches, the theory of fractals was also applied to the problem. Turk et al. (1987) spearheaded the calculation of the fractal dimension D for the 10 type-profiles using the divider method. A compilation of other fractal correlations is available in $\mathrm{Li}$ and Huang (2015). Additionally, Magsipoc et al. (2019) recently published an overview of most methods for surface roughness determination in two and three dimensions that have been applied also in part to JRC determination. Till now, correlating all kinds of mathematical parameters with JRC, using different techniques is ongoing. Besides many others, for example, Ficker and Martišek (2016) used an image recognition approach, Wang et al. (2017) used support vector regression to calculate JRC, Yong et al. (2018) applied a vector similarity measure and Gravanis and Pantelidis (2019) employed the theory of random fields. However, to date, they remain singular cases and statistical and fractal means have been explored more often. Nevertheless, the usefulness of repetitive correlation of already used or new measures with JRC solely based on the type-profiles must be doubted for various reasons.

Within previous works, differences concerning the database exist. Even though all correlations refer to the 10 typeprofiles they were digitised by the various researchers in different ways, resulting in varying resolution and subsequently different sampling intervals. For example, the pioneers Tse and Cruden (1979) enlarged the profiles with a factor of 2.5 and picked points in an interval of $1.27 \mathrm{~mm}$ using a digitising table. Consequently, an effective sampling interval of approximately $0.5 \mathrm{~mm}$ was achieved. Yu and Vayssade (1991) and Tatone (2009) also performed scanning, enlarging, and manual tracing of the type-profiles at sampling intervals between 0.5 and $2.4 \mathrm{~mm}$. Instead, Bae et al. (2011) used a digital copy of the type-profiles for image analysis in order to extract points on the profiles automatically. They reported a resolution of 20 pixels per $1 \mathrm{~mm}$ resulting in a sampling interval of $0.05 \mathrm{~mm}$. Overall, concerning the digitalisations of the type-profiles, Tatone (2009) stands out. He was the first to make his digital type-profiles publicly available. Unfortunately, since then only Li and Zhang (2015) and Stigsson and Mas Ivars (2019) followed the lead and published their source data.

Besides the differing database, uncertainty concerning the calculation procedures exist. Often, the implementations of the algorithms and the pre-processing steps of the data are not described in their entirety. However, adequate traceability is especially important with fractal approaches. If thorough documentation is not supplied, reproduction of prior findings is nearly impossible or at least achieving similarity is, in either case, cumbersome. Consequently, even when the same data is used different values of JRC result. Especially for the fractal dimension, the discrepancies are large since several calculation routines have been used in the past (c.f. Magsipoc et al. 2019). Moreover, in this context, confusion exists regarding the applicability of the different fractal calculation approaches with respect to the self-affine nature of rock discontinuity profiles.

Most importantly, for any arbitrary profile, irrespective of the particular calculation approach, the same JRC should result. This is a requisite because of the referencing of all correlations to the 10 type-profiles. Consequently, statistical and fractal approaches must yield the same JRC. However, previous publications used statistical or fractal techniques independently and never compared resulting JRC directly. Furthermore, they often focused on the type-profiles only. Nevertheless, it is extremely important to compare the approaches for a larger dataset of profiles to evaluate their universal validity. Only Marsch et al. (2020) investigated the problem rudimentary. They showed that JRC calculated from the statistical parameter $Z_{2}$ were not equal or even satisfactorily similar to JRC calculated e.g. from the fractal compass dimension $D_{\text {comp }}$. Certainly, there is a strong need for analyses of naturally occurring profiles other than the type-profiles.

Due to the problems addressed above, this study aims towards testing the hypothesis whether for any profile the same JRC results independent of the calculation scheme. This is achieved by revisiting the type-profiles and additional application of the approaches to six different rock surfaces. Having particularly field engineers and practitioners in mind, the primary objective is to denominate the most simple, intuitive, and reliable approach amongst a selection of tools available, may they be statistical or fractal procedures.

Firstly, some general remarks on the quality and availability of the type-profiles are given. This is followed by a presentation of the mathematical methods used. In section four the sensitivity of these methods is discussed by evaluating different digitalisations of the type-profiles. Thereafter, 
the application of the approaches to six different rock samples forms the main part of the study. Finally, the outcome is discussed, and conclusions are drawn.

\section{General Issues Concerning the Data Base for JRC Correlations}

Without exceptions, the set of Barton and Choubey's (1977) ten type-profiles forms the basis for all previously published correlations between JRC and statistical or fractal values. As a marginal note - a few authors, e.g. Li and Zhang (2015) and $\mathrm{Li}$ et al. (2017), also included other profiles available in the literature, e.g. from Bandis (1980). However, this does not necessarily improve the correlations. When using these functions some fundamental problems with the referential type-profiles must be considered.

\subsection{Measurement Quality of the Original Type-Profiles}

Due to the only graphical presentation of the type-profiles, it is practically impossible to assess the quality of the measurements. Additionally, in the original publication the typeprofiles are downscaled compared to the initial measuring length whereby information is lost. Consequently, the profiles appear to be continuous which is incomprehensible, as the traces were gathered with a tactile profilometer and therefore should exhibit a stepwise progression (see Tatone and Grasselli 2010). Moreover, the device consists of aligned metal pins of usually $1 \mathrm{~mm}$ in diameter, which are pressed onto the surface to gather the height information. During this process, an unavoidable deformation of the pins occurs leaving behind small gaps in the trace and resulting in variable sampling intervals. Following the classic approach, the profilometer measurements are transformed to paper by hand, which adds another source of error as the pins might slip again. Additionally, with the apparatus roughness wavelengths below $1 \mathrm{~mm}$ are not resolved, and, obviously, wavelengths greater than the profilometer, which is usually 100 to $150 \mathrm{~mm}$ in length, are also not collected. Moreover, solely the largest amplitude over the length of the pin diameter is measured. The device, therefore, acts as a low-cut filter and band-pass filters the original surface trace (Maerz et al. 1990). Indeed, all measuring approaches let them be contactless optical devices or tactile procedures do have a limited resolution and low-cut filtering is always happening. However, with the tactile profilometer, the resolution is relatively low, compared to most recent measuring tools, amounting to $1 \mathrm{~mm}$ only.

In summary, due to the uncertainties described above it is pointless to use finer sampling resolutions when digitising the type-profiles (as it was done by many authors in the past). Consequently, applying more and more sophisticated measuring devices for natural surfaces with higher resolution is unneeded if the objective is to determine the JRC based on the 10 type-profiles. At all times, it must be kept in mind that all correlations rest upon the sparse, low-resolution, and from today's perspective inexact type-profiles.

\subsection{Available Input Data}

As pointed out in the introduction, only a hand full of researchers made their digitalisations accessible. As an example for the available three data sets, in Fig. 1 the fifth type-profile $(\mathrm{JRC}=9.5)$ is displayed. At first glance the traces appear similar. However, with increasing length the variations in height grow larger. There seems to exist a tipping point at approximately $32 \mathrm{~mm}$ where a steep increase in the lines is seen. After that, the lines diverge more. Certainly, due to contrasting digitalisation procedures discrepancies are visible and consequently statistical and fractal parameters will be, most likely, unequal amongst the data sets. Additionally, in Fig. 1 the overall trends of the traces are indicated as dotted lines colour-coded according to each originator. It will be shown that removing these overall trends is of major importance. It is essential to establish a horizontal datum line always in the same manner to obtain reproducible and reasonable roughness measures.

Apart from the height variations, different degrees of detail are also visible in Fig. 1. Especially between the blue line and the red and orange lines at the relative peak at $55 \mathrm{~mm}$ length, the contrast is large. Generally, the various authors of the available correlations not only used separate methods for digitalisation but also, applied disparate sampling intervals. This is also valid for the profiles in Fig. 1. Accordingly, the amounts of sampling points and the

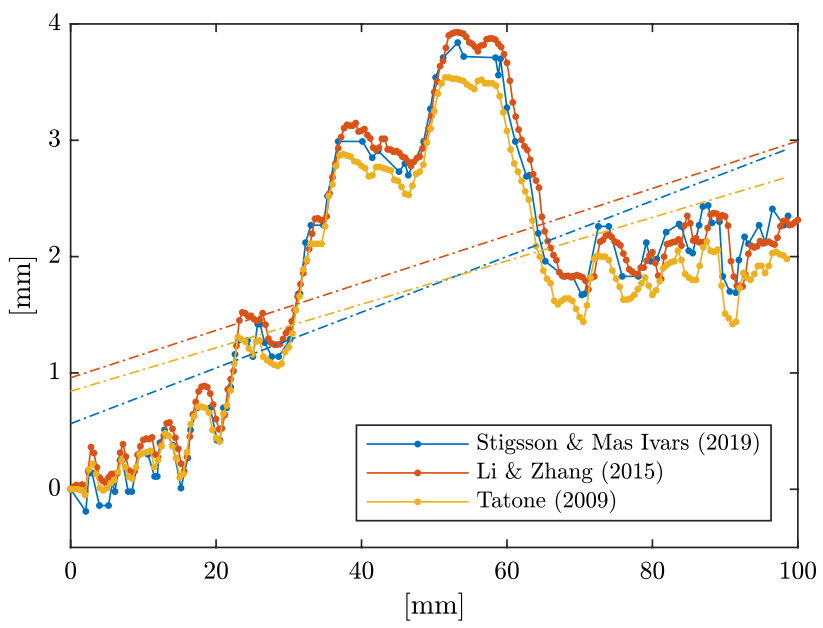

Fig. 1 Variation of the fifth type-profile $(\mathrm{JRC}=9.5$, exaggeration in height 15) 
sampling intervals are shown in Fig. 2. Li and Zhang (2015) and Tatone (2009) sampled the profiles with a constant interval of $0.4 \mathrm{~mm}$ and $0.5 \mathrm{~mm}$, respectively. Instead, Stigsson and Mas Ivars (2019) picked points with an average spacing of $1 \mathrm{~mm}$ using sampling steps of up to $4.4 \mathrm{~mm}$, however referencing their later correlation to a different, not available data set from Jang et al. (2014).

Remember, the original data from Barton and Choubey (1977) was gathered using a resolution of $1 \mathrm{~mm}$. Therefore, using a larger sampling rate would inevitably lead to noise in the secondary profiles. On the other hand, obviously using lower sampling rates would lead to a loss of information. Already Yu and Vayssade (1991) addressed the issue related to sampling. However, they only pointed out that it is important to adjust the correlation functions to the sampling interval and vice-versa. Regardless, for their (inaccessible) digitalisation they also used sampling intervals smaller than the one of the original type-profiles.

For future works (in case adding another correlation to the already plenty is desired) it is advisable to use an existing data set to eliminate an influencing variable. From the available data of Figs. 1 and 2, the one from Tatone (2009) is most suitable as a reference set: the type-profiles were digitised using the original sampling interval of $1 \mathrm{~mm}$ and the overall trends in the profiles have been removed. Moreover, his routine is well documented and good enough in light of the low graphical quality of the original type-profiles.

\section{Methods}

In principle, there are two possibilities to calculate JRC: using statistical parameters, or using fractal approaches in various peculiarities. In isolated cases, other approaches

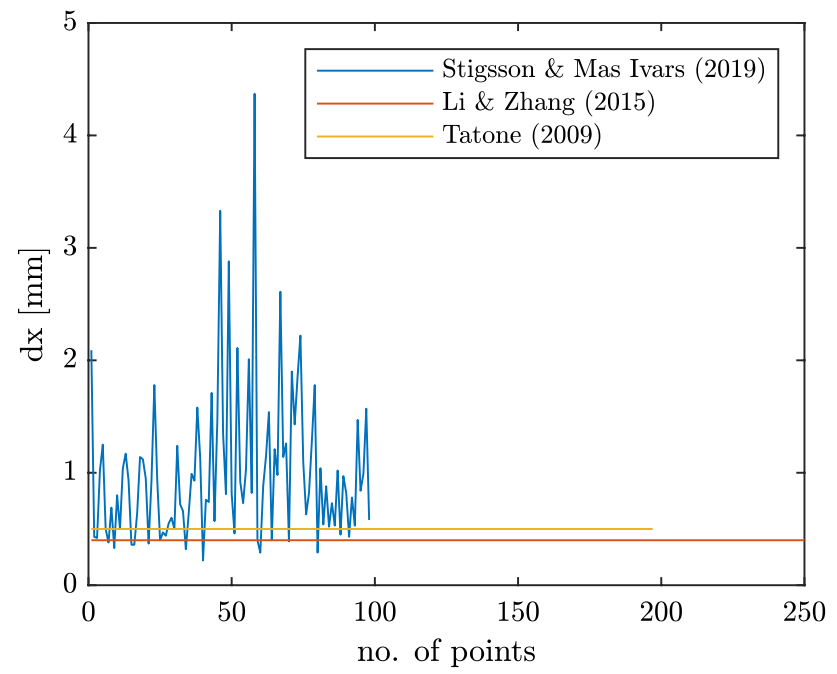

Fig. 2 Amounts and spacings of sampling points for type-profile five have been used (e.g. Tatone 2009; Zhang et al. 2014; Pickering and Aydin 2016). The mathematical implementation of most of the statistical parameters is straightforward and software for simple spreadsheet analysis suffice for the calculation. Instead, certain algorithms for the calculation of the fractal dimension are quite elaborate, mathematically complex, and consume computational power. For this paper, all calculation approaches were implemented in Matlab@ and the function scripts and data are available in the online repository to this publication (see Marsch 2020).

\subsection{Statistical Parameters}

From all statistical parameters that have been used in rock mechanics the root mean square of the first derivative of the surface profile, named $Z_{2}$, is the one used most (cf. Li and Zhang 2015). Myers (1962) established the parameter for use in material sciences and it is written in Eq. 1. When using a constant sampling interval Eq. 1 evolves to the discrete form of Eq. 2 where $N$ is the total number of vertices and $y_{\mathrm{i}}$ the height coordinate of the profile points.

$Z_{2}=\sqrt{\frac{1}{L} \int_{x=0}^{x=L}\left(\frac{\mathrm{d} y}{\mathrm{~d} x}\right)^{2} \mathrm{~d} x}$

$Z_{2}=\sqrt{\frac{1}{(N-1)(\mathrm{d} x)^{2}} \sum_{i=1}^{N-1}\left(y_{\mathrm{i}+1}-y_{\mathrm{i}}\right)^{2}}$

Besides, individual authors used other statistical parameters, such as the standard deviation of the slope angles of each sampling step $\sigma_{\mathrm{i}}$ (Yu and Vayssade 1991) or the ultimate slope of the profile $\lambda$, as a measure of the peak amplitude of the profile versus its projected length (Barton and de Quadros 1997). Examples of other parameters used more often than $\sigma_{\mathrm{i}}$ and $\lambda$ are the roughness profile index $R_{\mathrm{p}}$ (Tatone and Grasselli 2010; Yu and Vayssade 1991; Maerz et al. 1990) and the so-called Structure Function of the profile, termed SF (Tse and Cruden 1979; Yu and Vayssade 1991; Yang et al. 2001). $R_{\mathrm{p}}$ is defined as the ratio between the actual versus the projected length of the profile whereas SF can be calculated from $Z_{2}$ by introducing the sampling interval.

It will be shown later that the statistical parameters mentioned are interchangeable to a certain degree. Therefore, in this study, the inference of JRC from statistical values is represented by using $Z_{2}$ and the correlation from Tatone and Grasselli (2010). Indeed, other correlations, such as the ones from Yu and Vayssade (1991) or from Tse and Cruden 1979, yield quite similar results. However, Tatone and Grasselli (2010) made their input data accessible and they used a 
$1 \mathrm{~mm}$ sampling interval for digitalisation in compliance with Barton and Choubey (1977). The correlation of JRC with $Z_{2}$ from Tatone and Grasselli (2010) reads as follows:

$\mathrm{JRC}_{Z_{2}}=55.03 \cdot\left(Z_{2}\right)^{0.74}-6.1$

\subsection{Fractal Measures}

By means of the theory of fractals, it is possible to measure irregular and complex natural patterns even on small scales (Hastings and Sugihara 1994). The essential requirement for the use of the fractal concept for roughness determination is the distinction between (a) self-similarity and (b) self-affinity. These qualities exist for natural entities that are build out of recurring patterns of themselves so that they look alike and/or have the same statistical properties on different scales. With self-similar profiles, rescaling is isotropic. However, for self-affine profiles rescaling has to be different for each direction to obtain the same appearance and/or statistical properties. Kulatilake et al. (2006) postulated that rock discontinuity profiles are of self-affine nature. This is fundamental since the difference between self-similarity and self-affinity entails the need for diverse calculation procedures for the fractal dimension $D$ or the Hurst-exponent $H$, which convert into each other.

In past studies, the term fractal dimension has been used quite unthinkingly in the context of rock roughness evaluation. Of course, different authors applied different calculation procedures, e.g. compass walking, box counting or spectral analysis. Consequently, different numbers for $D$ for the type-profiles were calculated (e.g. Lee et al. 1990; Odling 1994). However, researchers claimed to have calculated "the fractal dimension". Instead, it is of paramount importance to distinguish between the values according to the calculation algorithms, as they are obviously not interchangeable. Mandelbrot (1985) himself-who is widely regarded as the initiator of the fractal theory-suggested that different denominations, such as "compass dimension" or "box dimension", should be used.

Concerning fractal measures, in the beginning of their application to JRC calculation, researchers like Turk et al. (1987) and Lee et al. (1990) used compass walking and the divider method. Later, Den Outer et al. (1995) and/or Kulatilake et al. (2006) have identified these approaches as being inadequate in capturing the self-affine nature of rock profiles. Nonetheless, this did not prevent the use of these approaches thereafter (e.g. Bae et al. 2011). Moreover, the impracticality of the methods has not been shown thoroughly but was rather argued solely on a theoretical basis. Consequently, compass walking is the method used most frequently in the context of roughness evaluation (cf. Li and Huang 2015). Apart, correlating the compass dimension of the type-profiles with JRC works just fine. Therefore, in this study compass walking is also analysed for comparative reasons.

In the already mentioned, most recent work by Stigsson and Mas Ivars (2019) an important fact is underlined: fractal calculation schemes are meaningful only if an additional magnitude parameter is incorporated. Magsipoc et al. (2019) recognized this simultaneously, identifying power spectral density and the root-mean-square correlation method (RMS$\mathrm{COR}$ ) as being eligible for roughness characterisation. Consequently, possible correlations of fractal measures with JRC need another parameter apart from $\mathrm{H}$ or $D$ to be fully constrained. Stigsson and Mas Ivars (2019) used RMS-COR and power spectrum analysis to produce $\mathrm{H}$ and an asperity measure, which they named $\sigma \delta h(\mathrm{~d} x)$. The two variables were then linked to the JRC. Accordingly, RMS correlation and spectral analysis are used in this study.

Of course, besides the three approaches used in this study and described hereafter, other fractal methods for roughness evaluation exist (for example box counting). However, they all lack reasonable correlation with JRC and are therefore discarded here. What is meant by "reasonable correlation" will become clear in the following and is discussed later.

$R M S$-COR method. With this method, the roughness of a profile is evaluated by using the variation of the distribution of height differences in dependence of spatial wavelength (Renard et al. 2006). For varying vertex intervals, $\mathrm{d} v$, a population of corresponding height differences, $\mathrm{d} h$, is acquired as depicted in Fig. 3. Then, the standard deviation of the height differences, $\sigma(\mathrm{d} h)$, for each vertex interval is calculated. By plotting $\sigma(\mathrm{d} h)$ versus $\mathrm{d} v$ in $\log -\log$ space the Hurst exponent is received as the slope of the linear fit. A requirement for this technique is that the profile vertices are equally spaced. Additionally, the method rests upon height

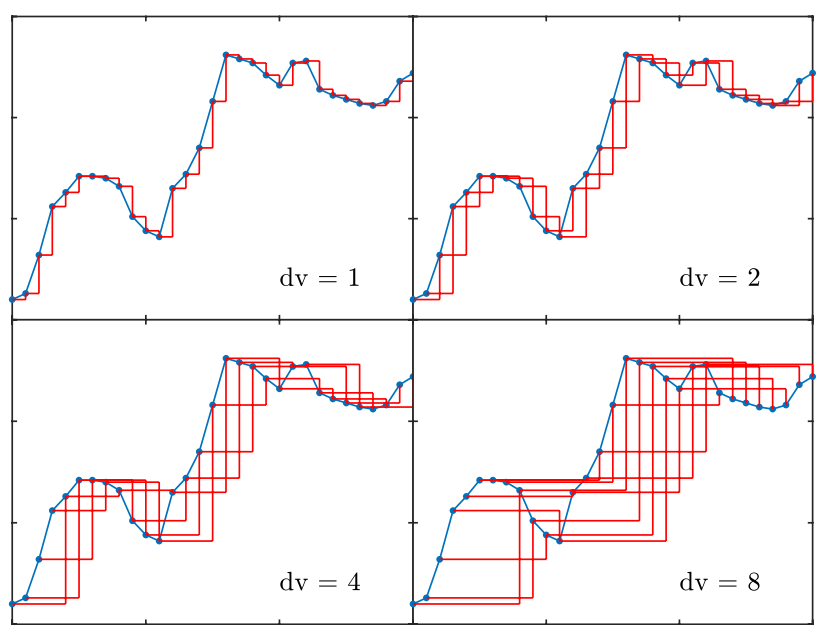

Fig. 3 Concept of RMS-COR method 
differences; therefore, the profiles must be free of possible overall trends.

An important aspect of the RMS-COR method is that the intercept of the linear fit line with the $y$-axis serves as a magnitude parameter for the profile. This value is necessary since the Hurst exponent only does not describe self-affine fractal entities distinctively (Magsipoc et al. 2019). In this study, the terminology from Stigsson and Mas Ivars (2019) for the magnitude parameter $\sigma \delta h(\mathrm{~d} x)$ is used. Also, they introduced a correlation of $H$ and $\sigma \delta h(\mathrm{~d} x)$ with JRC which is given hereafter:

$\mathrm{JRC}=-4.3+54.6 \cdot \sigma \delta h(1 \mathrm{~mm})+4.3 \cdot H$

Equation 4 is valid for sampling intervals of $1 \mathrm{~mm}$. As stated earlier, this should be the default for JRC determination. However, in case a different constant sampling interval $\mathrm{d} x$ is used $\sigma \delta h(1 \mathrm{~mm})$ evolves from scaling $\sigma \delta h(\mathrm{~d} x)$ according to the following conversion:

$\sigma \delta h(1 \mathrm{~mm})=\frac{\sigma \delta h(\mathrm{~d} x)}{(\mathrm{d} x / 1 \mathrm{~mm})^{H}}$

Furthermore, Stigsson and Mas Ivars (2019) stated that a shortcoming of the RMS-COR technique is an underestimation of $\mathrm{H}$ due to the natural finite length of profiles in general. To account for this effect, they constructed artificial profiles with pre-set Hurst exponents and asperity measures using an inverse Fast-Fourier-Transform algorithm. Subsequently, calculating $H$ with the RMS-COR method yielded systematically lower Hurst exponents for profiles having a generated $H$ of larger than 0.5 . They applied a rule for compensation, however, not including it in their writing. Therefore, adopting their approach and data, the following correction equation was produced in this study, being valid for the RMS-COR method and being necessary in case the calculated Hurst exponent, $H_{\mathrm{RMS}, \mathrm{cal}}$, is larger than 0.5:

$H_{\mathrm{RMS}}=\ln \left(H_{\mathrm{RMS}, \text { cal }}\right)+1.18$ for $H_{\mathrm{RMS}, \text { cal }}>0.5$

Power spectrum analysis. Predominantly in tectonophysics power spectral analysis using the Fast-Fourier-Transform (FFT) is often applied for the evaluation of roughness of rock joints and faults (e.g. Candela et al. 2009; Bistacchi et al. 2011; Corradetti et al. 2017). FFT algorithms convert the spatial information of the profile to the frequency domain. By plotting the associated power versus each length frequency in log-log space, the Hurst exponent is received as the slope of the linear fit. Additionally, the asperity measure $\sigma \delta h(\mathrm{~d} x)$ can be calculated using $H$ and the intercept with the ordinate of the linear fit together with an evolution of sine waves according to the frequency spectrum of the profile (see Stigsson and Mas Ivars 2019). To calculate JRC Eq. 4 can be used which is also applicable to $H$ and $\sigma \delta h(\mathrm{~d} x)$ inferred from power spectral analysis.
As for the RMS-COR method, with spectral analysis discrepancies exist concerning the calculation of the Hurst exponent using FFT for fractal lines of known $H$. Here, an overestimation was seen and Stigsson and Mas Ivars (2019) introduced another equation for compensation purposes:

$H_{\mathrm{FFT}}=0.616 \cdot \ln \left(H_{\mathrm{FFT}, \mathrm{cal}}\right)+0.92$ for $H_{\mathrm{FFT}, \mathrm{cal}}>0.7$

Compass walking. The procedure is essentially an approximation of the length of a profile through a chain of circles in which each subsequent circle originates at the intersection of the previous circle with the profile. By repetition, a relationship is obtained between the various radii and the corresponding number of circles needed to cover the profile. Depending on the implementation, possible relics of the profile (parts not covered by circles) are neglected or incorporated in the analysis, the latter being valid for the algorithm that was implemented in this study. The slope of the linear fit to the data plotted in $\log -\log$ space yields the fractal compass dimension $D_{\text {comp }}$. A visualisation of the technique is given in Fig. 4.

The crucial setscrew in this approach is the compass radius. It is somewhat unclear which specific radii different authors used. However, Lee et al.'s (1990) provided some values. Coherently, their set of radii and their equation were used in this study. Their correlation reads as follows:

$\mathrm{JRC}=-0.87804+37.7844\left(\frac{D_{\text {comp }}-1}{0.015}\right)-16.9304\left(\frac{D_{\text {comp }}-1}{0.015}\right)^{2}$

A disadvantage of Eq. 8 is its quadratic, non-monotonic composition. The approach might be adequate for fitting the data of the type-profiles but if compass dimensions larger than 1.016738 are calculated erroneously low JRC would result (angular point of the equation). Therefore, the linear

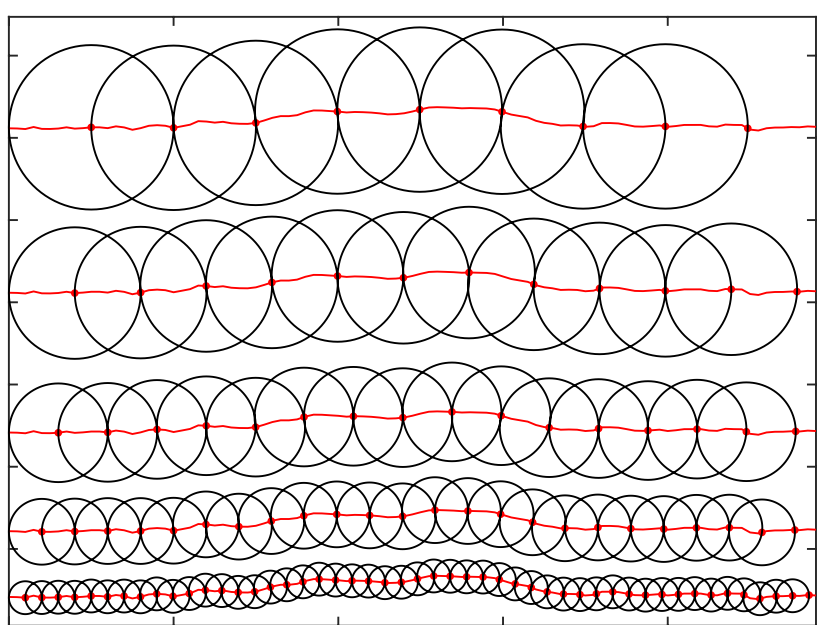

Fig. 4 Concept of compass walking method 
correlation from Turk et al. (1987) was also used. It is written in the following expression:

$\mathrm{JRC}=-1138.6+1141.6 \cdot D_{\text {comp }}$

\section{Re-Evaluation of the 10 Type-Profiles}

One of the main problems with the existing JRC correlations is the traceability of their creation. Often, neither the digitalisations of the type-profiles and pre-processing steps are given nor the input variables for the calculation of the specific roughness measures are revealed. Therefore, the importance of this information is demonstrated in the following by revisiting the type-profiles using the three available data sets and different input variables.

\subsection{Statistical Approaches}

There are at least 10 different statistical parameters that have been correlated with JRC (see Li and Zhang 2015). To greater or lesser extent, for the 10 type-profiles decent coefficients of determination were found for the specific parameters with JRC. On the other hand, for example, Wang et al. (2019) stated that $\sigma_{\mathrm{i}}$ behaves approximately the same as $Z_{2}$. Overall, $Z_{2}$ is well established and used often, therefore, in Fig. 5 its relations to the four statistical parameters mentioned above are shown. Apparently, $\lambda$ is not correlated with $Z_{2}$. The reason for this could lie in its rudimentary formulation in mathematical and physical terms, as it is only the ratio of the peak amplitude to the projected length of the
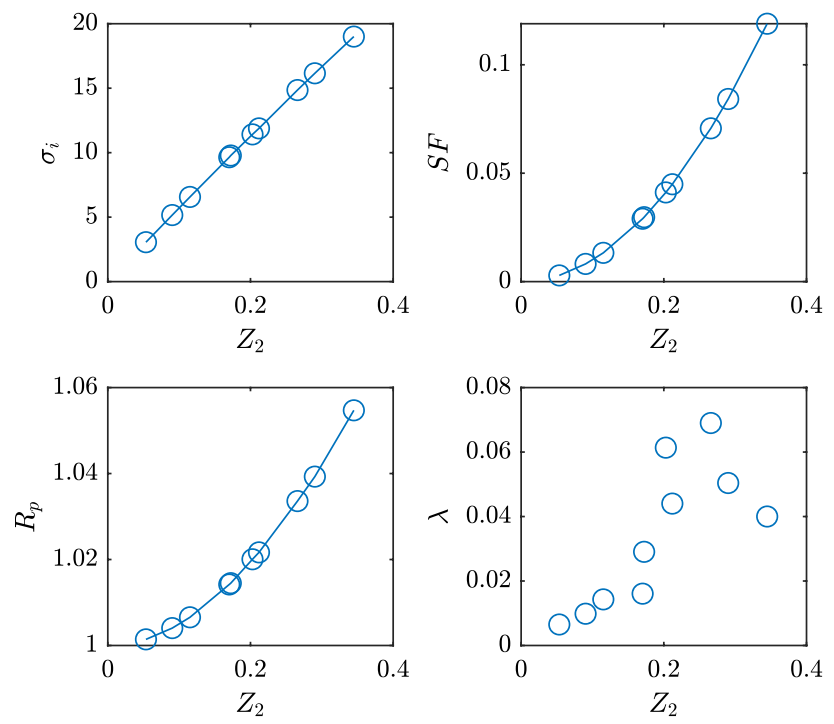

Fig. $5 \sigma_{\mathrm{i}}, \mathrm{SF}, R_{\mathrm{p}}$ and $\lambda$ versus $Z_{2}$ for the 10 type-profiles (profile data from Tatone 2009) profile. However, for $\sigma_{\mathrm{i}}, \mathrm{SF}$ and $R_{\mathrm{p}}$ there exist precise transfer functions with coefficients of determination of $R^{2} \approx 1$. The equations are given in Table 1 . Consequently, $\sigma_{\mathrm{i}}, \mathrm{SF}, R_{\mathrm{p}}$ and $Z_{2}$ are interchangeable and $Z_{2}$ can serve as an agent for the four statistical parameters.

Common sense implies more or less similar numbers for $Z_{2}$ regardless of the digitalisation of the type-profiles as long as the initial sampling interval is close to $1 \mathrm{~mm}$. However, this is not the case. In Table 2, the values of $Z_{2}$ exemplified again for the fifth type-profile according to each data set of Fig. 1 and the used equations (Eqs. 1 and 2) are given in the second and third column. Note, that it has been common practice to use only the discrete form of $Z_{2}$ as of Eq. 2. There exist no differences concerning the use of Eqs. 1 or 2 for the data from Tatone (2009) and Li and Zhang (2015). This is not surprising as for both data sets the original sampling interval is constant (as shown in Fig. 2). However, for Stigsson and Mas Ivars (2019) data a clear contrast is calculated. In order to use Eq. 2 the data has to be equally spaced and their original data was interpolated according to the average sampling interval of $1 \mathrm{~mm}$.

As stated earlier, Barton and Choubey (1977) employed a $1 \mathrm{~mm}$ sampling interval, which should therefore be the default for all JRC calculations. Additionally, $Z_{2}$ is a parameter that relies on the calculation of height differences between adjacent points. Hence, a horizontal datum line has to be established. Accordingly, in Table 2 the values for $Z_{2}$ are given also for the case that the trend is removed and that subsequently the rotated profile is interpolated at $1 \mathrm{~mm}$ sampling steps. The trend removal was achieved by performing a linear least squares regression and rotating the profile by the resulting overall slope angle towards the horizontal axis

Table 1 Transfer functions for $\sigma_{\mathrm{i}}, \mathrm{SF}, R_{\mathrm{p}}$ and $\lambda$ to $Z_{2}$

\begin{tabular}{llr}
\hline Parameter & Transfer function & $\begin{array}{c}\text { Coefficient of } \\
\text { determination }\end{array}$ \\
\hline$\sigma_{i}$ & $\sigma_{i}=54.79 \cdot Z_{2}+0.25$ & $R^{2}=0.99$ \\
$\mathrm{SF}$ & $\mathrm{SF}=\left(Z_{2}\right)^{2}$ & $R^{2}=1.00$ \\
$R_{\mathrm{p}}$ & $R_{\mathrm{p}}=0.414 \cdot\left(Z_{2}\right)^{2}+0.0188 \cdot Z_{2}+0.9991$ & $R^{2}=0.99$ \\
$\lambda$ & $\lambda=0.0306 \cdot \ln \left(Z_{2}\right)+0.0885$ & $R^{2}=0.62$ \\
\hline
\end{tabular}

Table $2 Z_{2}$ for type-profile JRC $=9.5$

\begin{tabular}{lllll}
\hline Data source & \multicolumn{2}{l}{ Original data } & $\begin{array}{l}\text { Rotated and } \\
\text { interpolated }\end{array}$ & $J R C$ \\
& Eq. (1) & Eq. (2) & & (Eq. 3) \\
\hline $\begin{array}{l}\text { Stigsson and Mas Ivars } \\
\quad \text { (2019) }\end{array}$ & 0.2577 & 0.1866 & 0.1840 & 9.6 \\
$\begin{array}{l}\text { Li and Zhang (2015) } \\
\text { Tatone (2009) }\end{array}$ & 0.2073 & 0.2073 & 0.1758 & 9.1 \\
\hline
\end{tabular}


referred to as "simple detrending" hereafter. In detail, this pre-processing procedure results in a decrease of $Z_{2}$ in the amount of 0.0315 for the data from Li and Zhang (2015) and 0.0213 for Tatone's (2009) data. These numbers are numerically small; however, it must be considered that this change is significant since the whole range of $Z_{2}$-values for all 10 type-profiles is only 0.4 . Consequently, the tailoring prior to calculation culminates in a change of $8 \%$ on the $Z_{2}$ scale. For the final JRC (far right column of Table 2) the variation decreases down to $4 \%$, however, a maximal difference of 0.5 on the JRC scale prevails.

Therefore, it is of vital importance to follow certain preprocessing steps vigorously, namely trend removal and $1 \mathrm{~mm}$ sampling, to receive comparable results for the same profiles from differing data sets. The effect of doing so is depicted for all type-profiles in Fig. 6. In part (a) and (b) it becomes clear that pre-processing reduces the values of $Z_{2}$. The transformation of $Z_{2}$ into JRC applying Eq. (3) is depicted in parts (c) and (d) of Fig. 6. For the uncorrected data, JRC in excess of 20 are calculated. This contradicts the range of the typeprofiles, which is marked with a rectangle in (c) and (d). Most importantly, correcting all data sets yields very good accordance with the curves and moves them to a reasonable range of JRC. This comparison leads to the conclusion that, if proceeded as mentioned above, analysing data originally gathered with sampling intervals close to $1 \mathrm{~mm}, Z_{2}$ is
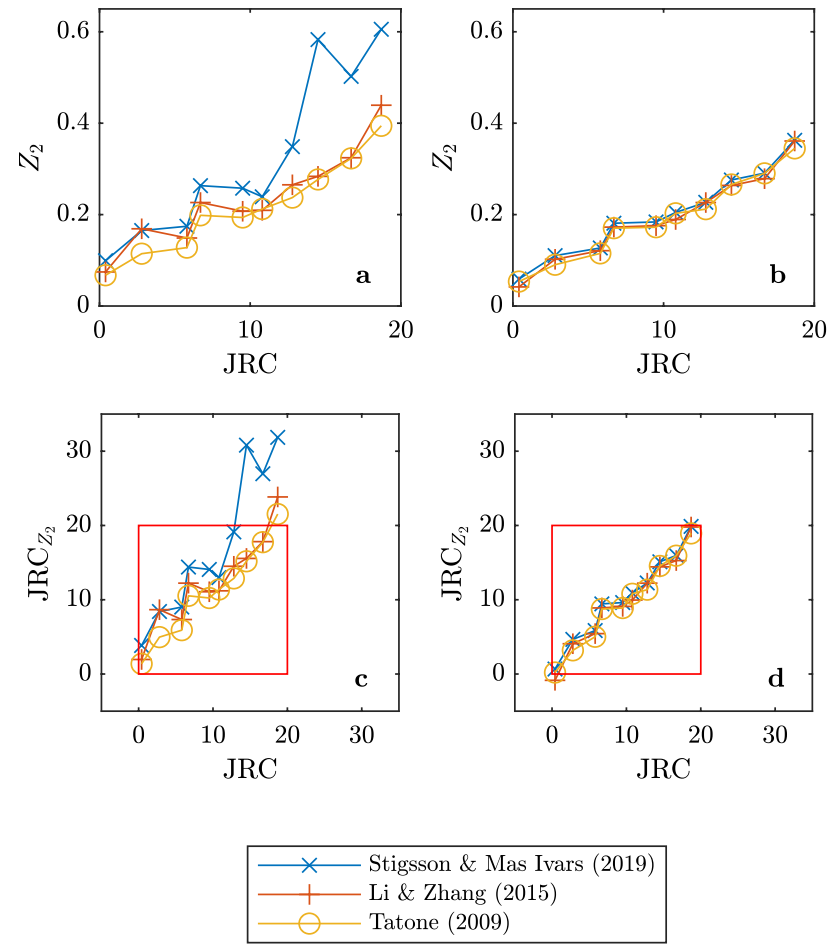

Fig. 6 Influence of the input data and pre-processing in the $Z_{2}$ approach and deduced JRC for the type-profiles; $\mathbf{a}$ and $\mathbf{c}$ original data, b and $\mathbf{d}$ simply detrended and interpolated insensitive to the input signal. This means that using $Z_{2}$ is a robust approach to calculating JRC.

\subsection{Fractal Approaches}

RMS-COR method. In Fig. 7 the relationships for $\sigma(\mathrm{d} h)$ and $\mathrm{d} v$ are plotted for the 10 type-profiles. In this case, all possible numbers for $\mathrm{dv}$ are shown, ranging from 1 to 100 for a $100 \mathrm{~mm}$ profile sampled at $1 \mathrm{~mm}$. It becomes visible that the progression of the curves is erratic for large vertex interval length $\mathrm{d} v$. To account for this unavoidable effect due to the natural finite length of the profiles, Malinverno (1990) proposed using only vertex intervals smaller than $20 \%$ of the total length $L$ of the trace (dotted-dashed line in Fig. 7). However, this number is too large to restrict all graphs to a more or less linear portion.

The influence of the vertex interval length on $H_{\mathrm{RMS}}$ and $\sigma \delta h(1 \mathrm{~mm})$ is illustrated furthermore in Fig. 8. The three cut-off length for $\mathrm{d} v$ from Fig. 7 are evaluated, namely 10 , 15 and $20 \%$ of the total length of the profile. For comparison, the results from Stigsson and Mas Ivars (2019) were re-digitised from one of their graphs. The largest differences, both for $H_{\mathrm{RMS}}$ and $\sigma \delta h(1 \mathrm{~mm})$, are seen for $\mathrm{d} v=0.2 \mathrm{~L}$. Concerning the case of $\mathrm{d} v=0.15 \mathrm{~L}$ good agreement is achieved for $H_{\mathrm{RMS}}$, however, for JRC larger than 15 differences occur for the asperity measure, $\sigma \delta h(1 \mathrm{~mm})$. Instead, a cut-off at a maximal vertex window of $10 \%$ of the total length excludes the first dents for most curves of Fig. 7 and results in a good agreement with the reference data for $\sigma \delta h(1 \mathrm{~mm})$ in Fig. 8 .

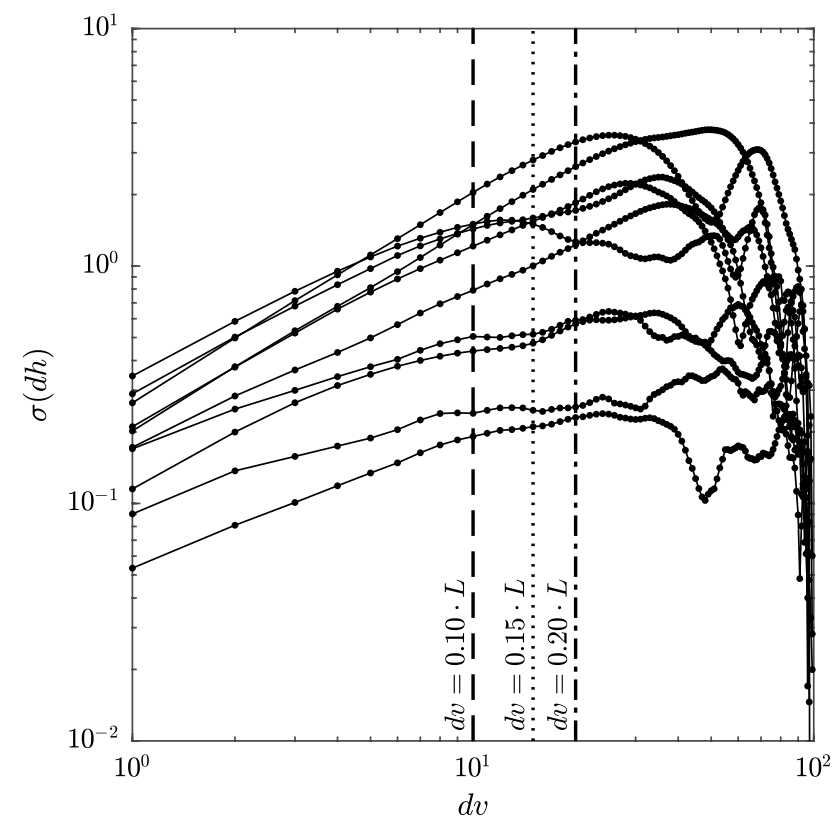

Fig. 7 Finite length effect regarding the RMS-COR method for the type-profiles (profile data from Tatone 2009) 

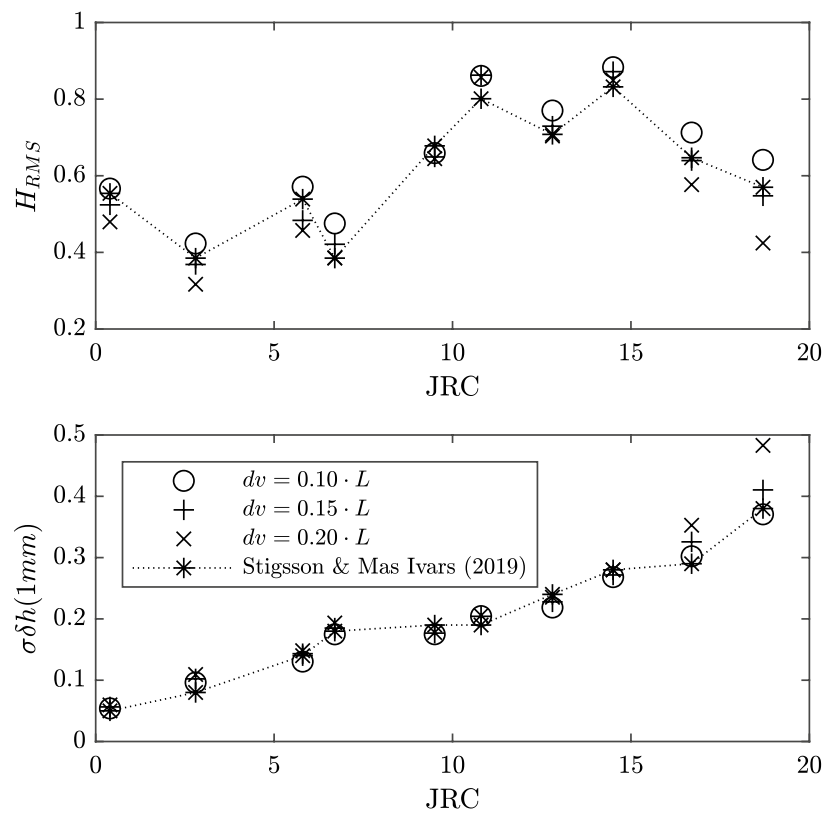

Fig. 8 Influence of vertex interval length dv in RMS-COR method for the type-profiles (profile data from Tatone 2009)

Taking into consideration both Figs. 7 and 8, in this study the vertex interval length $\mathrm{d} v$ was limited to $10 \%$ of the total profile length. This is reasonable regarding especially the relationship between $\sigma(\mathrm{d} h)$ and $\mathrm{d} v$ in Fig. 7. Consequently, it leads to acceptable Hurst exponents and asperity measures regarding the only available comparative values. The discrepancies can be explained by divergent input data and by the fact that it is somewhat unclear which cut-off value for $\mathrm{d} v$ Stigsson and Mas Ivars (2019) used and, subsequently, what values they included in their linear fit of $\sigma(\mathrm{d} h)$ and $\mathrm{d} v$ in $\log -\log$ space. Unfortunately, so far the procedure has not been fully described, considering all aspects necessary for reproduction. Therefore, in this study, the methodology applied has been fully disclosed.

As for the statistical measure $Z_{2}$ it is also important for the RMS-correlation method to assess its dependence on the data fed into the algorithm. From Fig. 9 it becomes clear that for all three data sets of the type-profiles $H$ and $\sigma \delta h(1 \mathrm{~mm})$ reach very similar values. Therefore, also RMS-correlation method is rather forgiving concerning imprecision during digitalisation. Note that the conclusions are drawn from detrended and interpolated input data, which is also a necessity for this technique as it operates with height differences of adjacent points on the profile.

Power spectrum analysis. For the calculation of JRC from power spectral analysis Eq. 4 can be used. However, it is important to realise that this correlation function was derived under consideration of only 64 vertices of each typeprofile. Stigsson and Mas Ivars (2019) argued that the input signal length for FFT processing should be a power of two.
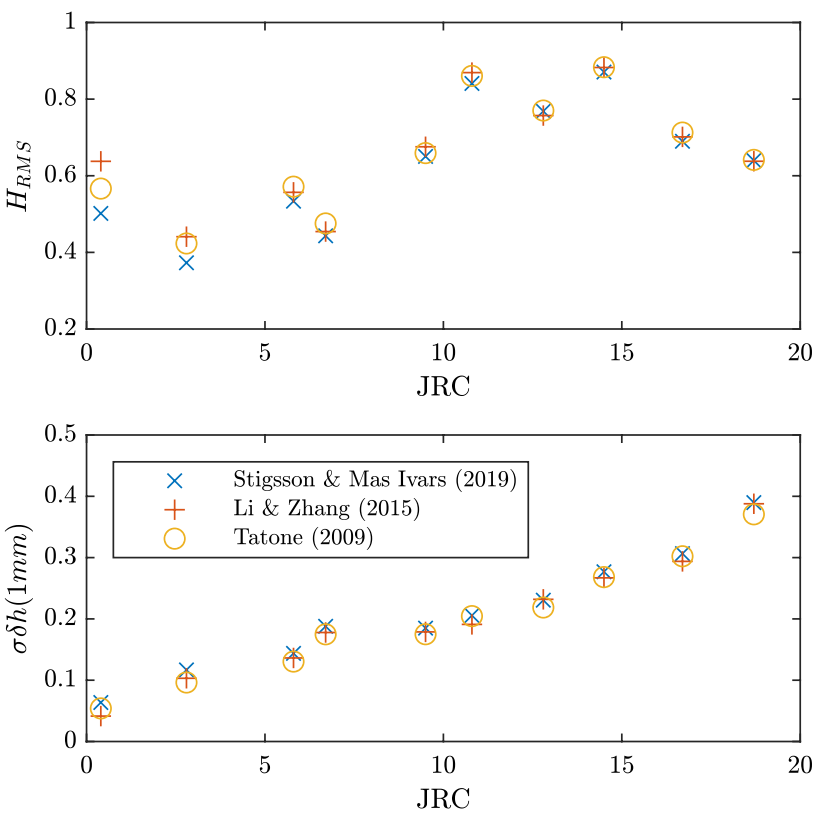

Fig. 9 Influence of the input data in the RMS-COR method for the type-profiles $(\mathrm{d} v=0.1 \mathrm{~L})$

In fact, it is only a question of speed of the particular algorithm: FFT is fastest for data lengths being powers of 2 and slowest for length values being prime numbers. However, most implementations of the FFT algorithm factorise the sample length thereby splitting up the transformation into manageable subsets (being necessary really only if large data is analysed).

In this study, Matlab@ was used in which the FFT is implemented according to Frigo and Johnson (2005). Their library is capable of dealing with whatever signal lengths and with non-periodic signals as well. Consequently, all points of the type-profiles can be considered. This is advisable since omitting $36 \%$ of data points in the already scarce population of the type-profiles produces large uncertainties.

Another issue in using Eq. 4 in conjunction with the spectral approach concerns the pre-processing of the input signal. Stigsson and Mas Ivars (2019) applied a "vertical adjustment [of the profile vertices] to avoid artificial high power biases of low frequencies". This vertical adjustment is essentially a proportional linear reduction of the $y$ coordinates according to the height difference over the full profile length. However, constricting the data in that way introduces a bias in the high-frequency range. Moreover, it directly effects the amplitude of the signal and therefore alters the asperity measure $\sigma \delta h(\mathrm{~d} x)$. From a practical point of view keeping pre-processing of the input signal to a minimum seems reasonable as it avoids the possible introduction of errors. The scope should be to implement simple and safe routines to infer JRC. Therefore, the type-profiles are re-evaluated using FFT but this time applying it to the same input signals 
as for the other methods. Consequently, all available data points from the type profiles are considered which were simply detrended and which were sampled coherently at $1 \mathrm{~mm}$ intervals.

The effects of simple detrending versus vertical adjustment are plotted in Fig. 10. By definition, Eq. 4 is suitable to produce JRC from spectral analysis and from RMS-correlation. This implies that more or less equal numbers for $H$ and $\sigma \delta h(1 \mathrm{~mm})$ should result irrespective of the two methods. For the case of vertical adjustment of the input signal, depicted in (c) and (d) of Fig. 10, noteworthy differences evolve compared to the values generated with RMS-COR (dashed line in Fig. 10). However, attention must be payed to the fact that in order to reproduce Stigsson and Mas Ivars (2019) data for FFT the first ten segments of a $100 \mathrm{~mm}$ profile containing 64 vertices have to be analysed and from that population, the one with the maximal asperity measure is
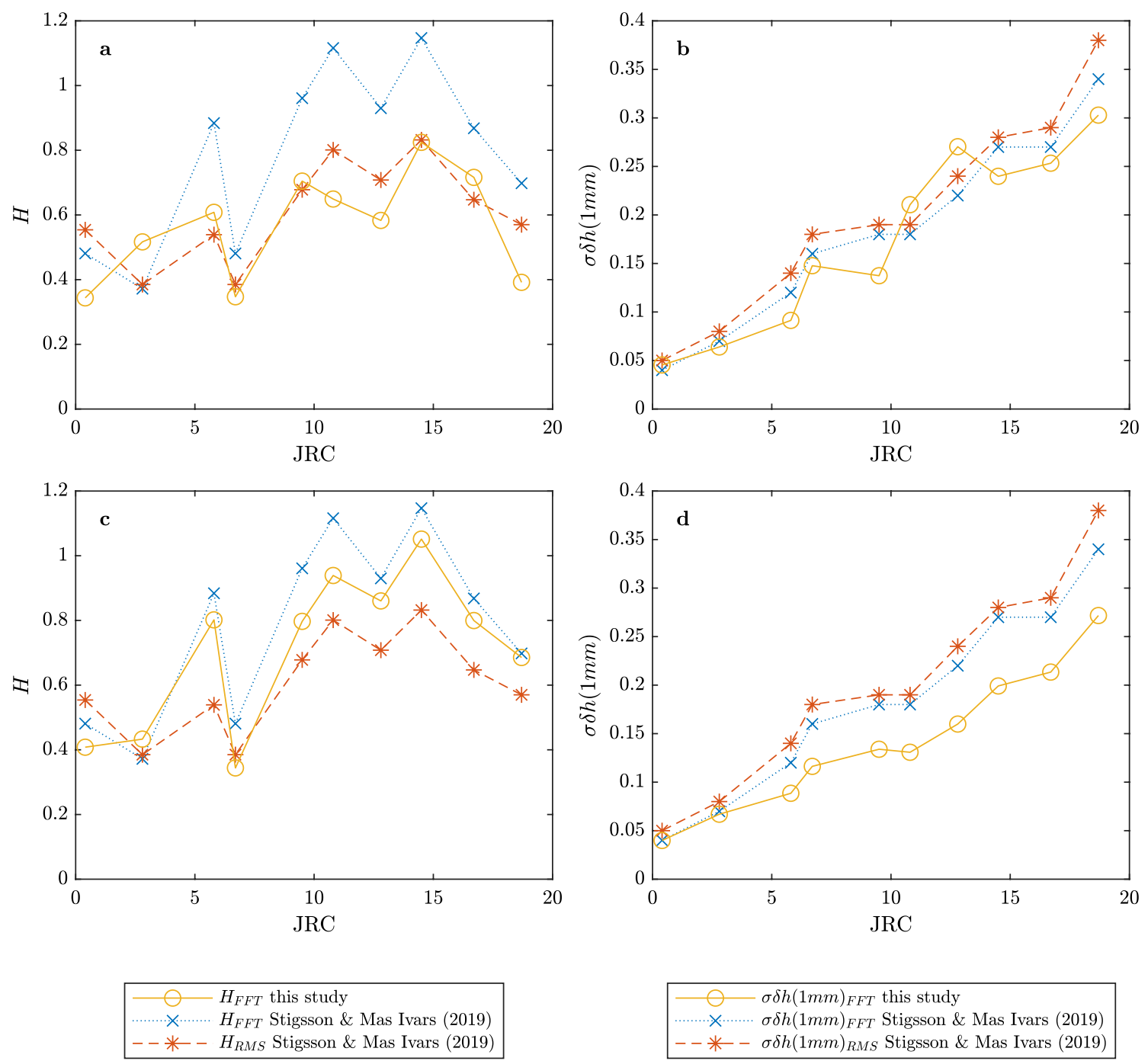

considered representative. However, in case the same and full, simply detrended input signal is used for FFT and RMSCOR, better agreement between the two methods is seen as in (a) and (b) of Fig. 10. Most importantly, since the heights of the profile vertices are not tampered with the asperity measure accords to an acceptable degree for both methods, as visible from (b) compared to (d) of Fig. 10. Consequently, when the whole length of the type-profiles $(100 \mathrm{~mm})$ is considered compensation as of Eqs. 6 and 7 is not necessary in order to obtain similar results with FFT and RMS-COR.

In fact, the introduction of the compensation Eqs. 6 and 7 presupposed that using an inverse Fast-Fourier-Transform (and consequently a spectral approach) is acceptable to produce the reference profile. Indeed, RMS-COR is a different mathematical algorithm relying on height differences between points and, therefore, it leads to divergent results. However, the finite length effect can be coped with by

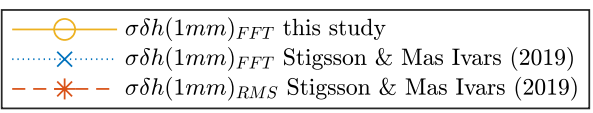

Fig. 10 Influence of pre-processing on $H$ and $\sigma \delta h(1 \mathrm{~mm})$ calculated using FFT: a and b simple detrending, $\mathbf{c}$ and $\mathbf{d}$ vertical adjustment (profile data from Tatone 2009) 
limiting the vertex interval length $d v$ to 10 percent of the profile length, as explained earlier, and a "correction" of $H$ as of Eq. 6 seems to be not necessary. In case of Eq. 7 the situation is even more inconsistent. From a theoretical point of view, compensation should be unnecessary, however, for a fractal line, which is produced using an inverse FFT, the forward application of that algorithm results in different values of $H$. Once more, these contradictions illustrate the complexity of fractal approaches.

To enable comparison of the calculation methods also the sensitivity of the power spectrum analysis to the input is investigated. The calculated values for $H$ and $\sigma \delta h(\mathrm{~d} x)$ are depicted in Fig. 11. The differences in the asperity measure are small. However, for the Hurst exponent even the datasets from Li and Zhang (2015) and Tatone (2009) show considerable differences although they have similar constant sampling intervals. Therefore, compared to $Z_{2}$ and RMSCOR methods the spectral approach is sensitive to the input signal.

Compass walking. With this method, the pivotal choice to be taken involves the set of radii. The upper part (a) of Fig. 12 shows the recalculated compass dimensions for the type-profiles for the case that Lee et al.'s (1990) set of radii is used $(r=2,4,6,8,10 \mathrm{~mm})$ and for the case, Turk et al.'s (1987) parameter set $(r=2,6,20,60 \mathrm{~mm})$ is used. Additionally, another set of radii containing five values spaced equally on a logarithmic scale from 1 to $15 \mathrm{~mm}$ was introduced. It must be emphasized that although the latter set is perfectly reasonable, as its values are larger than the sampling interval of the profile, smaller than the profile length
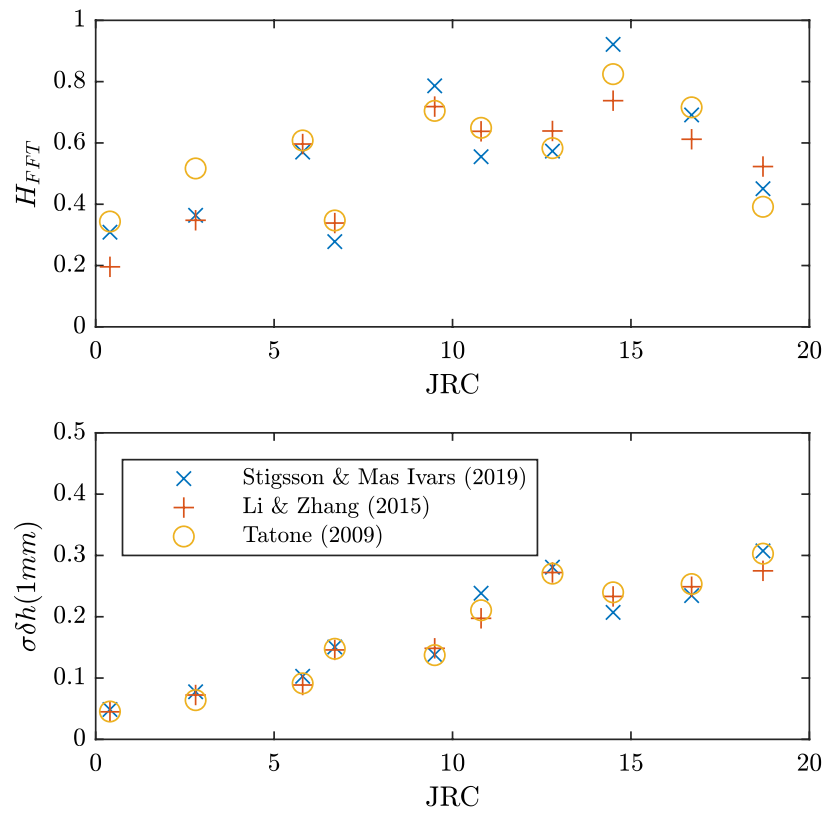

Fig. 11 Influence of the input data in the power spectrum method for the type-profiles (simple detrending) and it falls in the range of prior works, large differences occur. For type-profile 7 discrepancies in excess of 0.007 points on the scale of $D_{\text {comp }}$ result. This transfers into an enormous contrast of 9.6 for the JRC when Eq. 8 is used (part b) of Fig. 12) and amounts to 8.2 JRC-points when Eq. 9 is utilised (part c) of Fig. 12). For the other typeprofiles also divergence appears according to the set of radii used. Consequently, the technique is sensitive to the choice of compass radii. Furthermore, Fig. 12 illustrates the dependence of the compass walking method on the input data as, obviously, other profile data than the original from the authors of the equations was used (in this case from Tatone (2009)). Finally, irrespective of the three different sets of radii applied here, in general, with both correlations an underestimation of JRC emerges as most points lie beneath the 1-1 line (part b) and (c) of Fig. 12).

Overall, the fractal dimension determined by compass walking is in either case disputable for rock profiles. Especially the question of defining meaningful compass radii made Schmittbuhl et al. (1995) to advise against the use of this method. Regardless, the method functions well in the sense that $D_{\text {comp }}$ correlates with JRC for the type-profiles. Therefore, for comparative reasons and due to its broad use, compass walking is also assessed in this study. In order to being able to make use of Lee et al.'s (1990) correlation, coherently, in this paper, their set of radii, namely $r$ equalling $2,4,6,8,10 \mathrm{~mm}$, is used.

\section{Application to a Dataset of Natural Surface Traces}

Most information mentioned afore refer to the type-profiles. In case specific prerequisites are appreciated, exercisable techniques exist to closely calculate the given JRC values from the geometry of the profiles. Obviously, as all correlations reference to the "same" 10 type-profiles very similar JRC are determined irrespective of the calculation method. But is this also valid for non-standard profiles? In this section, that question shall be elucidated by analysing profiles extracted from three-dimensional models of natural rock surfaces.

\subsection{Data Acquisition and Handling}

To produce the surface models the rock samples were scanned using a GOM ATOS structured light scanner. The resulting representations feature a volumetric average RMS of residuals of $40 \mu \mathrm{m}$ based on having scanned spherical markers under the same conditions and within the object space of the rock samples. In Table 3, the resolution of each sample is listed. The device used is a highly capable metrology system that can be considered 

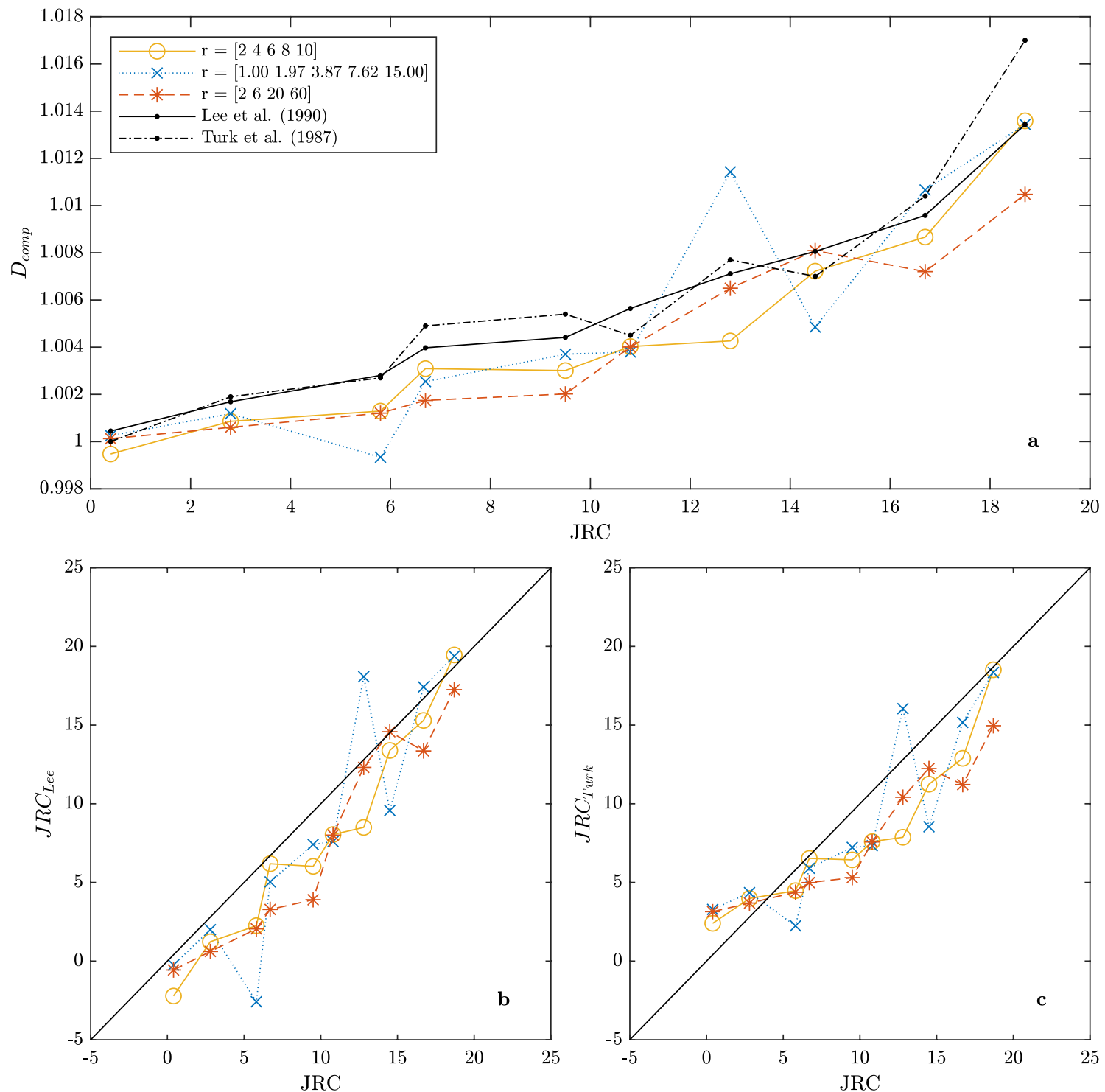

Fig. 12 Influence of the radii on the compass dimension $D_{\text {comp }}$ and JRC (profile data from Tatone 2009)

Table 3 Samples used in this study

\begin{tabular}{llll}
\hline Sample & $\begin{array}{l}\text { Model resolution [pts/ } \\
\mathrm{mm}^{2} \text { ] }\end{array}$ & Length/width [mm] & $\begin{array}{l}\text { Category according to } \\
\text { ISRM (1978) }\end{array}$ \\
\hline Basalt, B & 47.6 & $178 / 113$ & I-rough stepped \\
Granite, G & 51.3 & $172 / 95$ & II-smooth stepped \\
Limestone, K & 60.5 & $170 / 116$ & IV-rough undulating \\
Schist, SF & 55.0 & $170 / 103$ & V-smooth undulating \\
Sandstone, SI & 55.2 & $162 / 112$ & VII-rough planar \\
Sandstone, SS & 53.2 & $166 / 104$ & IV-rough undulating \\
\hline
\end{tabular}

a standard in the industry. According to Marsch et al. (2020), consequently, the surface models produced with this procedure are trustworthy and useful in rock roughness evaluation.
Also, in that prior publication, a routine was established in which all possible standard-length profiles on a surface mesh can be extracted. In the procedure, which is also applied in this study, height information is sampled 
with the sliding window method, simulating an imaginary profilometer of $100 \mathrm{~mm}$ length that traces the mesh in a user-defined raster, in this case, $1 \mathrm{~mm} \times 1 \mathrm{~mm}$ whereas the actual sampling interval amounts to $0.1 \mathrm{~mm}$. By doing so, a large quantity of profiles can be gained. All profiles are preprocessed individually (trend-removal) and then evaluated according to the methods explained earlier. This course of action is reasonable since it overcomes the usual randomness of measuring only one profile that is then said to be representative for a surface. Moreover, scale effects are avoided as the length of the extracted profiles conforms to the length of the type-profiles.

\subsection{Rock Samples}

Depending on the formation regime rocks develop different textures and consequently different roughness characteristics. Hence, upon evaluation of the universal validity of a theory, it is advisable to consider samples from all three main rock classes, namely igneous, metamorphic, and sedimentary rocks. As in this study, statistical and fractal calculation routines are compared, that are solely based on geometry, it is unimportant which specific rock material is used if the selection of surfaces covers the whole range of possible JRC from 0 to 20 . Consequently, the full bandwidth of roughness profiles starting with smooth appearances and ending at rough progressions must be analysed.

For this study, a collection of quarry stones was gathered to then manually introduce fresh tensional fractures into the rock blocks. That way the fracturing process is very much constrained and controllable. The resulting surfaces were presented to a group of five engineering geologists which were asked to select denominations according to the ISRM (1978). The roughness categories were assigned considering the whole laboratory size sample and therefore depict the small-scale roughness or unevenness. The average subjective assessments are given in Table 3. Note that the classification is by no means objective and the list shall only give a general impression of the surfaces. For a closer look and further personal analysis, the surface meshes are also accessible in the repository to this study (see Marsch 2020).

\subsection{Results}

The inference of JRC from statistical and fractal parameters obviously involves a mathematical transformation. As discussed earlier, the correlation functions incorporate assumptions and findings of the particular authors, which consequently add another level of complexity and uncertainty. Therefore, as a first step, the plain statistical and fractal values are examined.

Analysing the rough limestone sample K, in Fig. 13 the standardised histograms of the statistical parameters $Z_{2}, \mathrm{SF}$,
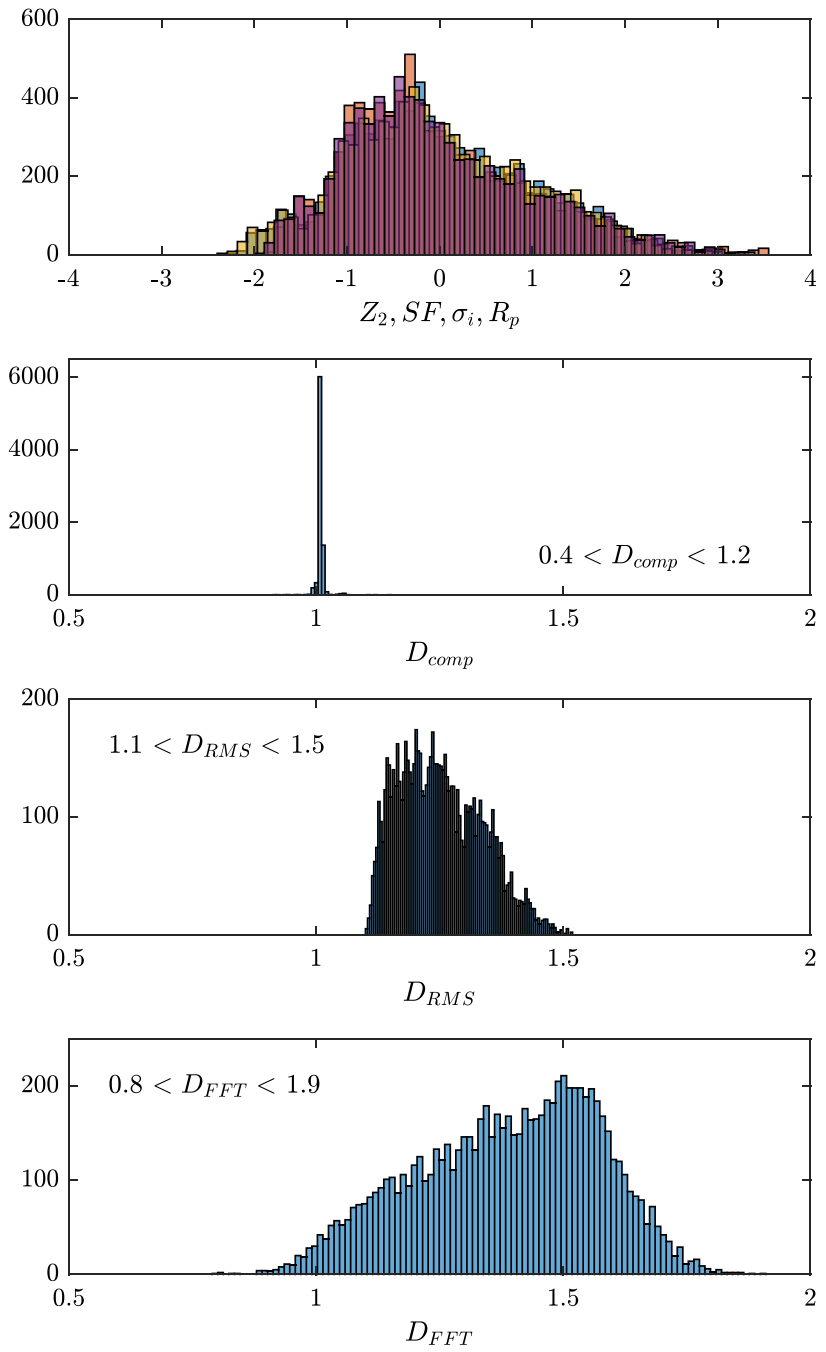

Fig. $13 z$-Scores of the statistical values and histograms of the fractal approaches, exemplified for sample $\mathrm{K}$

$\sigma_{\mathrm{i}}, R_{\mathrm{p}}$ and the distributions of $D$ according to the three methods for the determination of the fractal dimension are given. Note that the fractal distributions of course have not been standardised since they are theoretically of the same unit and scale. As expected, the $z$-scores for the four different statistical parameters are quite similar. Consequently, as a general approximation, these measures are also interchangeable just like for the type-profiles. However, concerning the fractal approaches, large discrepancies become visible. The compass dimension $D_{\text {comp }}$ exhibits a colossal peak at around one but shows a large range of values due to extreme outliers. Hence, compared to the other methods, the compass dimension is rather indistinctive on the scale of $D$ as it concentrates around unity. Yet, for roughness profiles, according to the theory, the fractal compass dimension should lie between one and two (cf. Hastings and Sugihara 1994). Indeed, profiles from more or less homogenous planer smooth surfaces 
would yield a low compass dimension of close to one, however, sample $\mathrm{K}$ has an undulating rough appearance. Taking a closer look at the values of $D$ from the RMS-correlation method and the spectral analysis leads to the conclusion that they do not accord. In agreement with the theory, the values of $D_{\text {RMS }}$ are very well situated between 1.1 and 1.5 . Instead, $D_{\text {FFT }}$ shows a wider range and unrealistic values below unity. These facts gain importance since the same correlation function (Eq. 4) is suggested to be used for both methods to infer JRC. In general, the distribution of the fractal dimension $D$ is evidently very much dependent on the specific determination routine. Additionally, when comparing the statistical with the fractal histograms no definite similarity of the distributions can be found. Most importantly, not only for sample K, but also for all other samples used in this study the findings above are valid.

Remember, as a requisite due to referencing of all correlations (statistical or fractal) to the 10 type-profiles, for any other profile as well, irrespective of the particular calculation approach, the same JRC should result. Consequently, JRC from fractal approaches versus JRC from statistical approaches ought to plot on the bisecting line. For all samples used in this study, these graphs are given in Fig. 14. Here, the inference of JRC from statistical approaches is represented by using $Z_{2}$, plotted on the abscissa.

There is some information that can be generalised from all samples. First, the fractal JRC values calculated according to Lee et al.'s (1990) Eq. 8 using $D_{\text {comp }}$ show a large divergence from equality with the statistical JRC. The variation is enormous and always covers more than the regular range of JRC values of 0 to 20. Hereby Eq. 8 delivers JRC smaller than zero regardless of the ISRM roughness category of the particular surface. Secondly, concerning the JRC inferred from spectral analysis, the graphs are diverse. For the undulating smooth sample SF and the planar rough sample, SI smaller discrepancy with the statistical JRC is seen compared to the other samples (still, the JRC values do not accord well). Apparently, the spectral analysis works better with surfaces that do not show large height differences or, stated otherwise, that show low variation and low amplitudes. Conversely, this underlines the critical issue within the spectral analysis of defining the asperity measure. By any means, the variation of $\mathrm{JRC}_{\mathrm{FFT}}$ is large and spans a range of at least $12.5 \mathrm{JRC}$-points (sample SS). Lastly, JRC

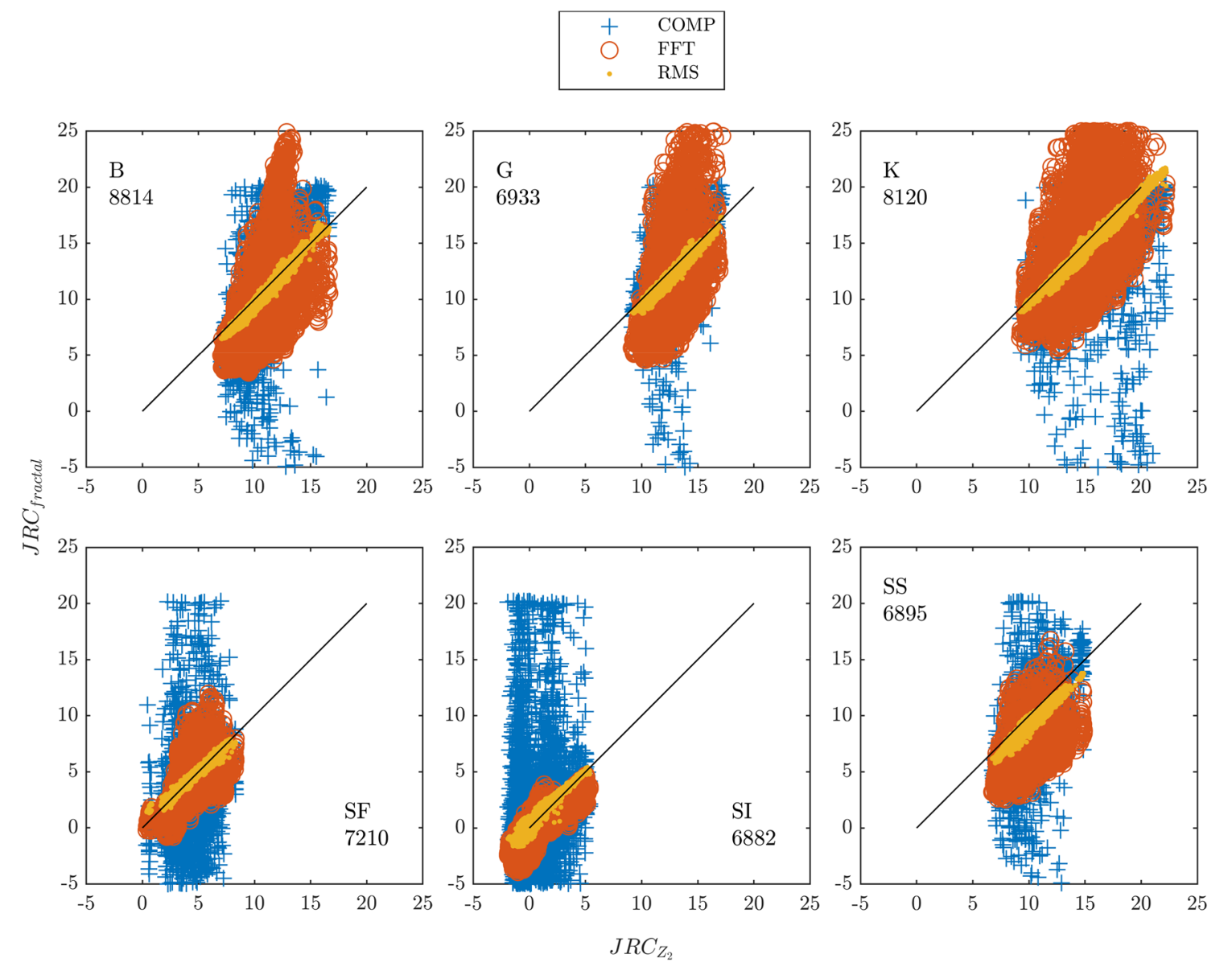

Fig. 14 Statistical versus fractal JRC (inset: sample name and total number of profiles analysed), simply detrended profiles 
determined with the RMS correlation method coincide with $\mathrm{JRC}_{\mathrm{Z2}}$. For practical application, the divergence from the 1-1 line is negligibly small. This applies to all samples. Notice here that the data were not transformed (Eqs. 6 and 7) following the results of the re-evaluation of the type-profiles.

Regarding only the type-profiles it was shown before that simple detrending is sufficient also for the use of the spectral analysis method to achieve acceptable results. However, the large variation of $\mathrm{JRC}_{\mathrm{FFT}}$ in Fig. 14 suggests that this is not the case for profiles other than the type-profiles. Especially in samples $B, G$ and $K$ large steps are present. Consequently, extracted profiles that incorporate these steps are discontinuous in the sense that the height difference between the ends of the profiles is large. This lack of periodicity will result in spectral leakage and therefore biased values for $H$ and $\sigma \delta h(\mathrm{~d} x)$ will be obtained. In order to eliminate this effect, the profiles were additionally vertically adjusted, and the outcomes are given in Fig. 15. Tapering the profiles removes almost all $\mathrm{JRC}_{\mathrm{FFT}}$ above the 1-1 line from the population (c.f. Fig. 14). However, the variation of JRC from spectral analysis remains large and the values bulge under the bisecting line. Consequently, this time the JRC is underestimated compared with the use of RMS-COR or $Z_{2}$. In case Eq. 7 was used to additionally reduce $H_{\mathrm{FFT}}$-values the differences would even increase. In summary, this underestimation is contradictive to the analysis of the type-profiles where the Hurst exponents based on the power spectrum systematically exceed the $H$-values from the RMS-COR method.

To make use of correlation functions for JRC in general, it was argued before that it is important to follow the procedures of the originators as close as possible. Therefore, a third case must be considered for using power spectral analysis with the six surfaces. As Stigsson and Mas Ivars (2019) used only the window of 64 vertices of the type-profiles with the largest asperity measure considering the first 10 vertices for their correlation, a similar procedure was used here. The rationale for this is that during direct shearing sample halves would most likely adhere to large steps of the surface. Therefore, these irregularities should be included in the roughness calculations. In this study, at first, the starting 65 vertices were taken from the $100 \mathrm{~mm}$ long profile. This window was then simply detrended and vertically adjusted and reduced to 64 points. Secondly, the procedure was repeated on the subsequent 65 vertices and so forth. Finally, the combination

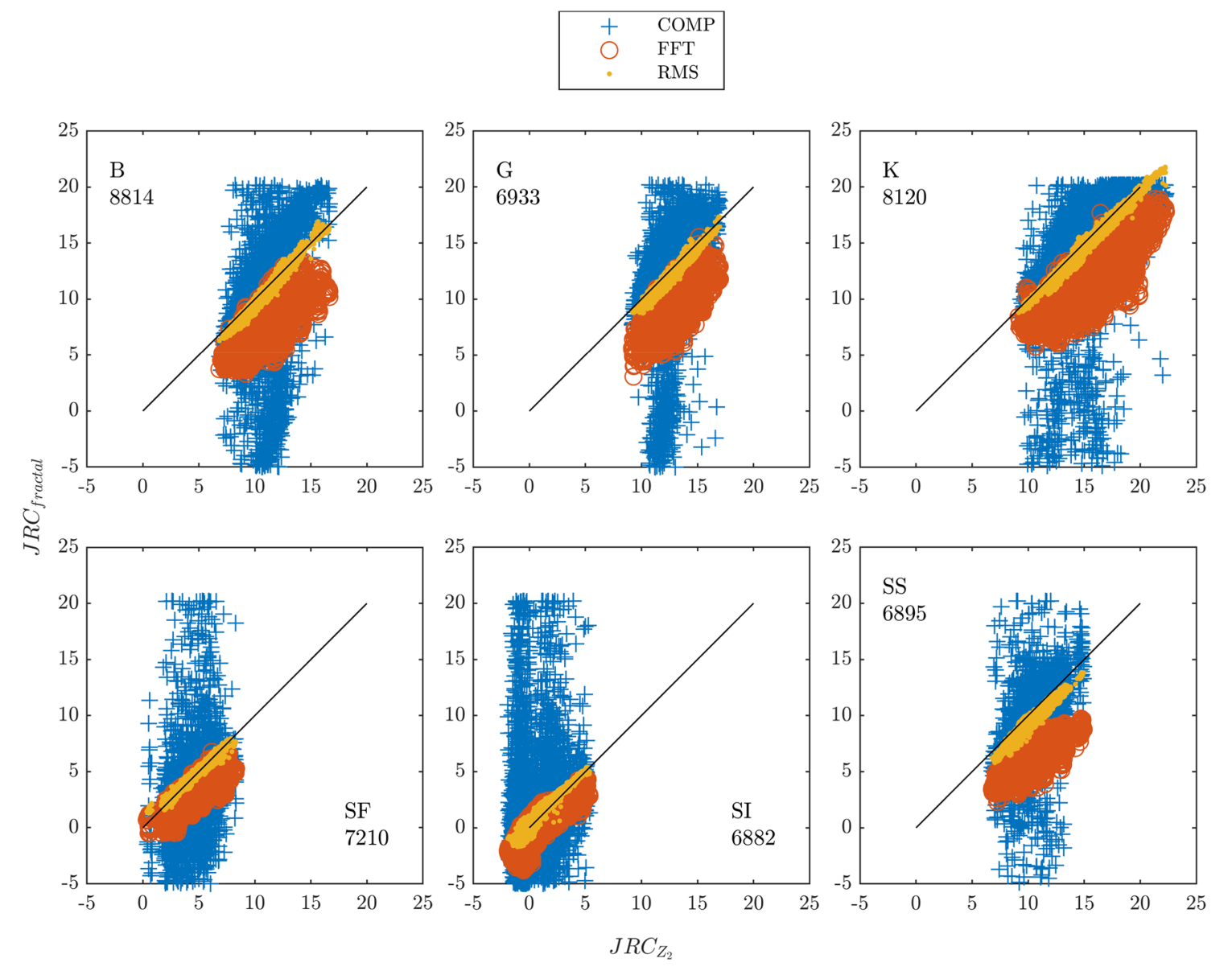

Fig. 15 Statistical versus fractal JRC (inset: sample name and total number of profiles analysed), vertically adjusted profiles 
of the Hurst exponent with the largest asperity measure from the group of 35 pairs was said to be representative for the standard-length profile. The results are shown in Fig. 16. For all samples, the values of $\mathrm{JRC}_{\mathrm{FFT}}$ were elevated compared to Fig. 15 and now gather around the bisecting line. However, the large variation persists and for sample $\mathrm{K}, \mathrm{JRC}_{\mathrm{FFT}}$ even extends to values greater than 20 . As for the two other cases, also with this processing divergent results are obtained upon the usage of spectral analysis.

However, the close agreement between JRC $\mathrm{RMS}_{\mathrm{ROR}}$ and $\mathrm{JRC}_{\mathrm{Z} 2}$ for all three pre-processing cases is somewhat striking since the RMS-Correlation method is rather approximative due to the need of linear regression of $\mathrm{d} v$ and $\sigma(\mathrm{d} h)$ in $\log -\log$ space. As stated earlier, fractal approaches need two parameters to be fully constrained. In Eq. 4 the share of the asperity measure $\sigma \delta h(1 \mathrm{~mm})$ in the final JRC is more than a factor of 10 larger than the share of the Hurst exponent. Consequently, the JRC calculated by RMS-correlation or spectral analysis predominantly depends on the magnitude parameter. Therefore, for a closer analysis $\sigma \delta h(1 \mathrm{~mm})$ is plotted versus $Z_{2}$ in Fig. 17. Most obviously, the scatter of $\sigma \delta h(1 \mathrm{~mm})$ deduced from the spectral analysis is large but instead, in the case of RMS-COR a higher degree of similarity with $Z_{2}$ is obtained. In fact, for the special case of analysing profiles with $1 \mathrm{~mm}$ sampling steps the two measures $\sigma \delta h(1 \mathrm{~mm})$ and $Z_{2}$ must be equal. The asperity measure is defined as the sample standard deviation of the population of height differences of adjacent vertices, $\delta h$, for a profile of $N$ points:

$\sigma \delta h(\mathrm{~d} x)=\sqrt{\frac{1}{N-1} \sum_{i=1}^{N-1}\left(\delta h_{i}-\overline{\delta h}\right)^{2}}$

A prerequisite for the calculation of all roughness measures was that a possible overall slope is eliminated. Any procedure of detrending should result in $\overline{\delta h}=0$. Consequently, Eq. (10) reduces to:

$\sigma \delta h(\mathrm{~d} x)=\sqrt{\frac{1}{N-1} \sum_{i=1}^{N-1}\left(y_{i+1}-y_{i}\right)^{2}}$

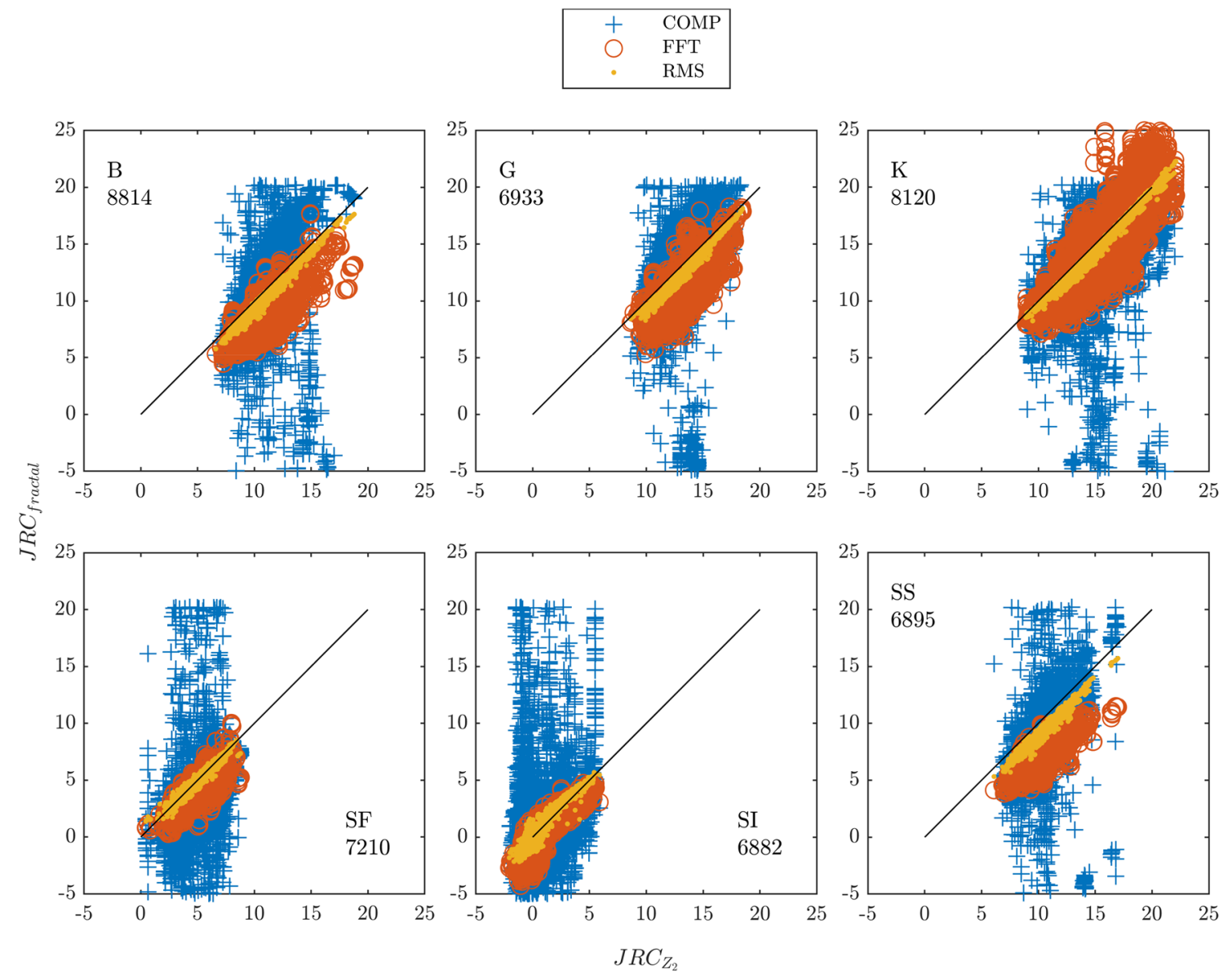

Fig. 16 Statistical versus fractal JRC (inset: sample name and total number of profiles analysed), vertically adjusted profiles and window of 64 vertices with largest $\sigma \delta h(1 \mathrm{~mm})$ per profile considered 

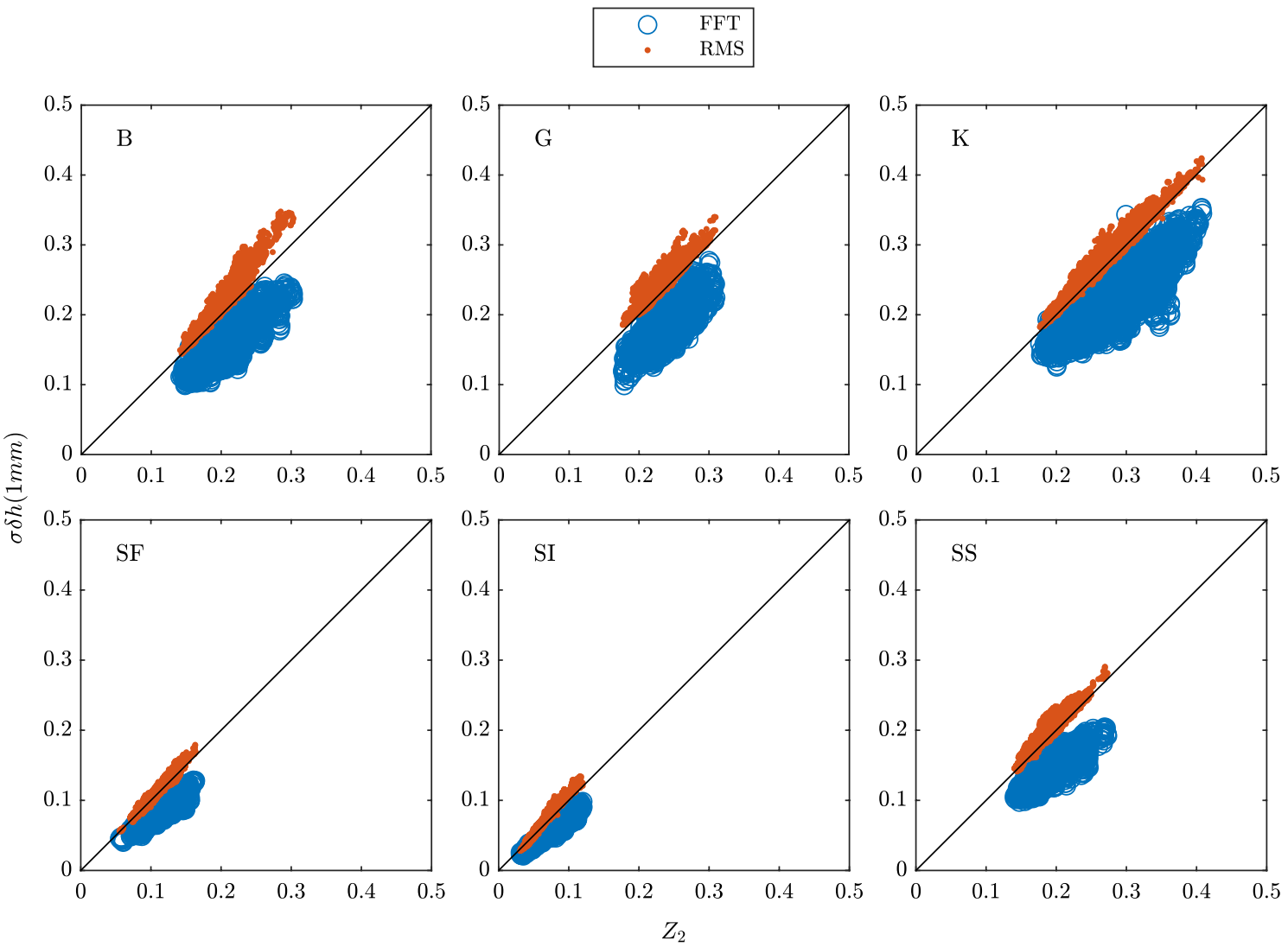

Fig. 17 Asperity measure $\sigma \delta h(1 \mathrm{~mm})$ versus $Z_{2}$, vertically adjusted profiles

Under consideration of Eq. 2 it becomes clear that $Z_{2}$ and $\sigma \delta h(\mathrm{~d} x)$ are linked by the inverse of the distance between the vertices, $\mathrm{d} x$, reading:

$Z_{2}=\frac{1}{\mathrm{~d} x} \cdot \sigma \delta h(\mathrm{~d} x)$

Now taking in mind that the concept of JRC estimation with the standard chart is valid for $1 \mathrm{~mm}$ sampling intervals only, in this particular case, $Z_{2}$ and the asperity measure are equal:

$Z_{2}(1 \mathrm{~mm})=\sigma \delta h(1 \mathrm{~mm})$

Consequently, all data points in Fig. 17 should lie on the 1-1 line. Indeed, $\sigma \delta h(\mathrm{~d} x)$ deviates from $Z_{2}$ for both RMSCOR and FFT methods. However, RMS-correlation surpasses the power spectrum approach in this matter.

\section{Discussion}

At first glance, it seems utterly simple to determine the JRC from profile traces: there exist the type-profiles as a reference along with some statistical or fractal parameters and subsequently, one can calculate the JRC for whatever profile. A closer second look however reveals many pitfalls: how good is the original database for the correlations anyhow? Are the working hypotheses comprehensible? Are the calculation methods useful and forward-looking? Finally, how can the results be evaluated, and, do they cover the whole data range of possible JRC adequately?

First, when dealing with the idea of determining JRC by correlation functions based on the type-profiles, the following must be considered: roughness increases from top to bottom in the standard chart. Consequently, the predicted JRC must increase. This apparently ordinary observation is in no way trivial: it makes it imperative that any mathematical parameter (or set of parameters) deduced from the profiles can be fitted by a monotonic function (or even better strictly monotonic). This irrevocable, paramount matter of fact is visualised in Fig. 18. As it is well-known, the statistical parameter $Z_{2}$ satisfies this requisite to the full extent without further input (cf. Fig. 6b)) whereas fractal approaches must yield at least two independent variables to meet this need (cf. Fig. 8). This study pointed out the close relationship of the asperity measure $\sigma \delta h(1 \mathrm{~mm})$ with $Z_{2}$. Indeed, these findings provoke the question of relevance concerning fractal approaches for JRC determination: why 


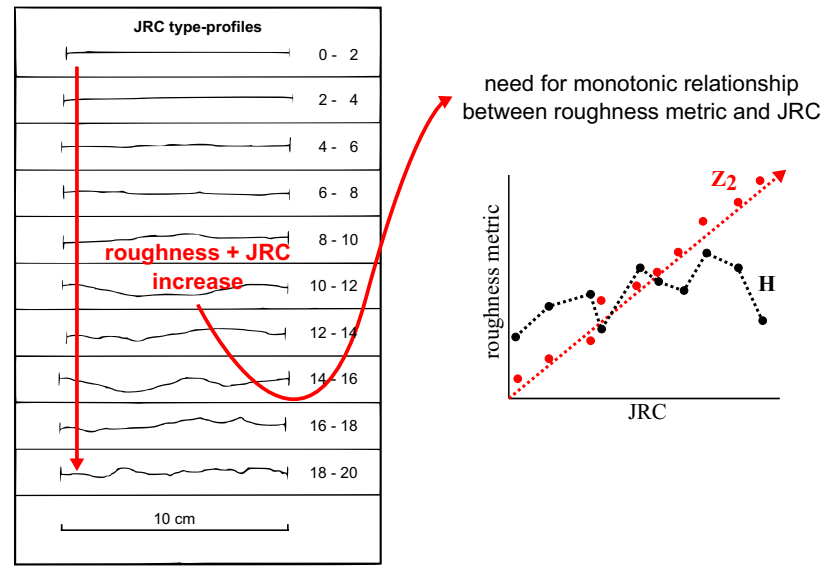

Fig. 18 Need for monotonic relationship (profile chart redrawn from Barton and Choubey 1977)

bother to calculate the Hurst exponent involving complex calculation routines to then scale that value with $\sigma \delta h(1 \mathrm{~mm})$ ? Using $H$ and $\sigma \delta h(1 \mathrm{~mm})$ produces unneeded sources of error and adds unnecessary ramifications. Moreover, the influence of $\sigma \delta h(1 \mathrm{~mm})$ on JRC in Eq. 4 is one magnitude greater than the input of H. Consequently, Eq. 4 is practically rather another statistical than a fractal correlation.

Undeniably, the visual nature of the type-profiles is of inferior quality. The information that can be gained from them is narrow. In fact, by revealing the effect of the sampling interval on JRC, Yu and Vayssade (1991) indirectly acknowledged the limitations of the standard chart. Therefore, re-digitalisation is useless and, at the latest, Tatone (2009), by sampling coherently in $1 \mathrm{~mm}$ intervals, squeezed out the best information. By far, his data suffices. Nevertheless, what was learned here from different data sets of the type-profiles is how sensitive specific calculation approaches are to the input signal. The robustness of the algorithms is absolutely essential before the background that it is impossible to exactly reproduce a measurement with simple tools like the tactile profilometer. Even using modern 3D scanners will not produce an identical image of a surface repeatedly. Nevertheless, if the measured object does not change then the roughness metric should not change either, at least not that much. Having this in mind $Z_{2}$ and RMS-COR clearly outperform the FFT routine as they yielded similar results using different digitalisations of the type-profiles.

In their original work, Barton and Choubey's (1977) stated that, preferably, JRC should be determined by direct shear testing. As a courtesy, they prepared the 10 type-profiles to provide engineers and geologists with a possibility to visually estimate JRC. However, over the past decades, opposed to the intended use of the type-profiles, researchers have driven correlating whatever measures with the typeprofiles to the absurd, claiming to be able to calculate JRC to decimal places based on re-digitising the original data over and over again. This led to a situation in which a confusing amount of correlations exists that each time refers to a very particular data set of the, although identical, typeprofiles. Moreover, divergent calculation schemes were applied and unfortunately, in most instances, they are poorly documented. Especially with fractal methods, however, it is extremely important to provide all variables to guarantee a safe application by others. Consequently, there is a need for standardised procedures.

A more general issue concerns the small quantity of the type-profiles. Establishing a universally applicable theory on 10 data points may result in an extreme simplification. Dealing with the natural, practically infinitely diverse matter, namely rock surfaces, demands a large database, at least to explore and prove the data distribution. Moreover, the application of extremely powerful algorithms to develop simple mathematical relationships based on so little input inevitably leads to overfitting. Additionally, there exist only a few datasets for cross-validation, of which some are given in Fig. 19. Certainly, for the type-profiles an acceptable relationship exists between JRC back-calculated from mechanical tests and JRC calculated from $Z_{2}$ or RMS-COR. At least, eight pairs lie near the 1-1 line. However, a considerable variation of more than five JRC-points is seen for the data from Bandis (1980), Bandis et al. (1983) and Grasselli (2001). Apparently, surfaces that have similar geometrical characteristics, resulting in similar $Z_{2}$ or $H$ and $\sigma \delta h(1 \mathrm{~mm})$ and consequently similar JRC, yield different shear strength. The same holds for the reversed statement. However, the reason for the poor distribution in Fig. 19 might lie in the selection of allegedly representative profiles from the surfaces, although, how this can be done objectively is beyond the scope of this study. Indeed, many different profiles could have been used as an agent for the surfaces in question (cf. Fig. 13).

In summary, relying on the type-profiles only and repetitive fitting of more and more parameters to them does not help the case. The problem of roughness estimation from geometrical information must be placed on a larger foundation. Consequently, openly available more significant, preferably three-dimensional geometric data accompanied by results from direct shear tests are needed.

\section{Conclusion}

In this study, considerable efforts were made to compare different methods for determining the JRC from roughness profiles and to make their differences transparent. This allows a direct comparison of statistical and fractal methods between each other which is a novelty in the field of JRC evaluation. The focus of this study was to verify the hypothesis whether for any arbitrary profile the same JRC results irrespective of 

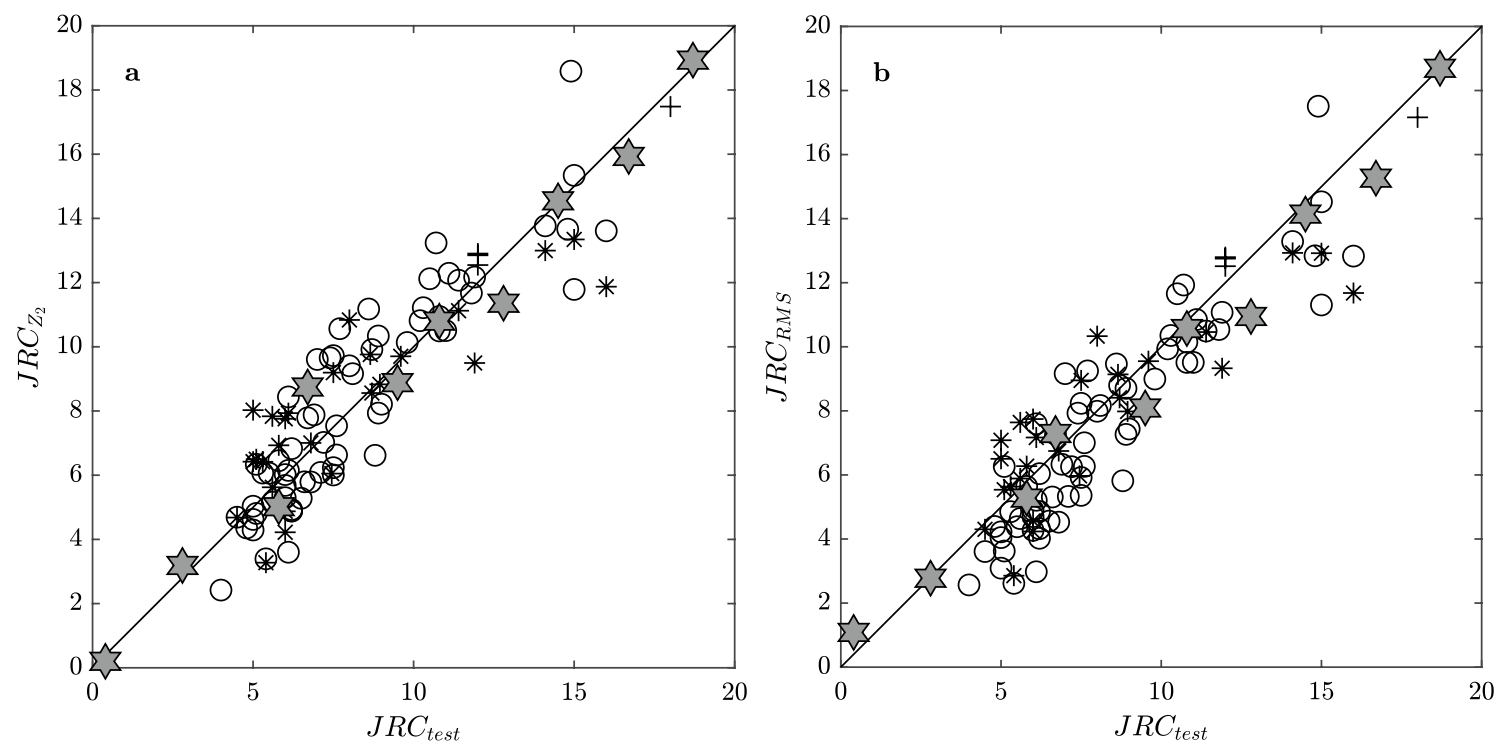

\begin{tabular}{cl}
$\bigcirc$ & Bandis (1980) \\
$*$ & Bandis et al. (1983) \\
+ & Grasselli (2001) \\
$\stackrel{\sim}{\sim}$ & Barton \& Choubey (1977) \\
\hline
\end{tabular}

Fig. 19 Relationship between back-calculated JRC and JRC inferred by geometrical means

using statistical or fractal approaches and to denominate the most reasonable practice.

To do so, first, problems concerning the input data for correlations, namely the initial type-profiles, were discussed. The sparseness and low-resolution of the original data bring about unquantifiable uncertainties. The application of more and more complex mathematical routines and extensive parametrisation is not appropriate. The type-profiles should not be considered the axiomatic definition of JRC, but they are still a smart tool for estimation.

On these grounds, secondly, the accepted state-of-theart mathematical methods to calculate reasonable statistical and fractal parameters have been described thoroughly, denominating and evaluating all input variables, and, thirdly, explaining their sensitivity by means of re-evaluating the type-profiles. All calculation routines and inputs used are available in the online repository to this publication (see Marsch 2020). Of course, disclosing every variable and pre-processing step is an absolute necessity for comparability and quality assessment. Thereby, the following can be concluded:

- $Z_{2}$ can be used as an agent for statistical approaches,

- the input signals must be detrended and interpolated at $1 \mathrm{~mm}$ intervals,

- a cut-off length with RMS-correlation of $\mathrm{d} v=0.1 \mathrm{~L}$ should be used, and

- spectral analysis is sensitive to the input data.
Obviously, for the type-profiles all methods lead to similar JRC values. Therefore, in the last step of this study, a vast number of profiles were extracted from different rock surfaces to investigate the interchangeability of the calculation schemes. Based on the analysis of these profiles the following statements stand:

- compass walking is inapplicable to rock traces,

- spectral analysis is defective concerning the profile-based concept of JRC, and

- RMS-correlation accords well with $Z_{2}$.

Concerning spectral analysis and RMS correlation, the inferred JRC heavily rely on the asperity measure. This applies to the approximation of JRC on 10-cm scale based on the type-profiles. However, clearly, the benefits of fractal approaches, being the determination of roughness on different scales and large areas, shall not be questioned.

Explicitly, thinking beyond the present type-profiles for JRC is advocated here. There exist to great uncertainty in Barton and Choubey's (1977) original chart. The type-profiles should only be used for what they have been initially introduced, namely approximating JRC. Reporting JRC to decimal places suggests an accuracy that does not exist. The best the user can obtain from the present correlations is the class of JRC.

Naturally, field engineers and practitioners want to apply routines safely. Therefore, offering suggestions on how to 


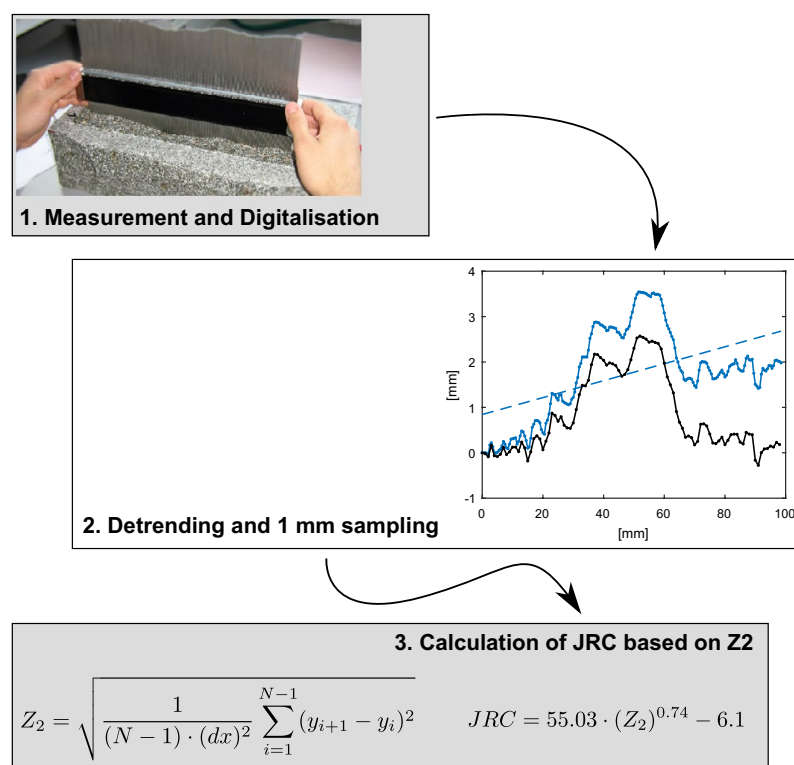

Fig. 20 Good enough, simple workflow

apply the concepts are required: using the simple measure $Z_{2}$ is good enough to determine the JRC from profiles as it is coherent with the well-meant, engineering-like standard chart. A suggested workflow for profiles of $10 \mathrm{~cm}$ length is given in Fig. 20. This is the best practice from a present perspective having only the sparse and low-resolution original type-profiles at hand for referencing.

A critical point in determining JRC based on two-dimensional data is most certainly the selection or identification of representative profiles. Indeed, Barton and Choubey's (1977) type-profiles are not objective as they have been chosen for unreproducible reasons: "an attempt was [...] made to select the most typical profiles". However, this issue was not discussed in this study deliberately since direct mechanical shear tests would have been necessary to calibrate possible new sampling routines. For the future, the question needs to be addressed of how representative and more inclusive two-dimensional geometric input data can be sampled from shear surfaces in case three-dimensional parameters are unwanted. This could lead to objective and better correlations with JRC.

Author contributions Both authors developed the concept of the study. Kristofer Marsch conducted the laboratory work, processed the data, and wrote the evaluation algorithms. Kristofer Marsch collected the literature for this study. Both authors interpreted the results. Kristofer Marsch wrote the manuscript, supported by suggestions by Tomas M. Fernandez-Steeger.

Funding Open Access funding enabled and organized by Projekt DEAL.
Availability of data and materials Link to repository: https://dx.doi. org/10.14279/depositonce-9592

\section{Compliance with ethical standards}

Conflict of interest There exist no conflicts of interest.

Open Access This article is licensed under a Creative Commons Attribution 4.0 International License, which permits use, sharing, adaptation, distribution and reproduction in any medium or format, as long as you give appropriate credit to the original author(s) and the source, provide a link to the Creative Commons licence, and indicate if changes were made. The images or other third party material in this article are included in the article's Creative Commons licence, unless indicated otherwise in a credit line to the material. If material is not included in the article's Creative Commons licence and your intended use is not permitted by statutory regulation or exceeds the permitted use, you will need to obtain permission directly from the copyright holder. To view a copy of this licence, visit http://creativecommons.org/licenses/by/4.0/.

\section{References}

Alameda-Hernández P, Jiménez-Perálvarez J, Palenzuela JA, El Hamdouni R, Irigaray C, Cabrerizo MA, Chacón J (2014) Improvement of the JRC calculation using different parameters obtained through a new survey method applied to rock discontinuities. Rock Mech Rock Eng 47:2047-2060. https:// doi.org/10.1007/s00603-013-0532-2

Bae D, Kim K, Koh Y, Kim J (2011) Characterization of joint roughness in granite by applying the scan circle technique to images from a borehole televiewer. Rock Mech Rock Eng 44:497-504. https://doi.org/10.1007/s00603-011-0134-9

Bandis S (1980) Experimental studies of scale effects on shear strength, and deformation of rock joints. PhD thesis, University of Leeds

Bandis SC, Lumsden AC, Barton N (1983) Fundamentals of rock joint deformation. Int J Rock Mech Min Sci Geomech Abst 20:249268. https://doi.org/10.1016/0148-9062(83)90595-8

Barton NR (1973) Review of a new shear-strength criterion for rock joints. Eng Geol 7:287-332. https://doi.org/10.1016/00137952(73)90013-6

Barton N, Choubey V (1977) The shear strength of rock joints in theory and practice. Rock Mech 10:1-54. https://doi.org/10.1007/BF012 61801

Barton N, de Quadros E (1997) Joint aperture and roughness in the prediction of flow and groutability of rock masses. Int J Rock Mech Min Sci 34:252.e1-252.e14. https://doi.org/10.1016/S1365 -1609(97)00081-6

Beer AJ, Stead D, Coggan JS (2002) Technical note estimation of the joint roughness coefficient (JRC) by visual comparison. Rock Mech Rock Eng 35:65-74. https://doi.org/10.1007/s006030200 009

Bistacchi A, Griffith WA, Smith SAF, Di Toro G, Jones R, Nielsen S (2011) Fault roughness at seismogenic depths from LIDAR and photogrammetric analysis. Pure Appl Geophys 168:2345-2363. https://doi.org/10.1007/s00024-011-0301-7

Candela T, Renard F, Bouchon M, Brouste A, Marsan D, Schmittbuhl J, Voisin C (2009) Characterization of fault roughness at various scales. Implications of three-dimensional high resolution topography measurements. Pure Appl Geophys 166:1817-1851. https ://doi.org/10.1007/s00024-009-0521-2 
Corradetti A, McCaffrey K, de Paola N, Tavani S (2017) Evaluating roughness scaling properties of natural active fault surfaces by means of multi-view photogrammetry. Tectonophysics 717:599606. https://doi.org/10.1016/j.tecto.2017.08.023

Den Outer A, Kaashoek JF, Hack HRGK (1995) Difficulties with using continuous fractal theory for discontinuity surfaces. Int J Rock Mech Min Sci Geomech Abst 32:3-9. https://doi. org/10.1016/0148-9062(94)00025-X

Ficker T, Martišek D (2016) Alternative method for assessing the roughness coefficients of rock joints. J Comput Civ Eng 30(4):4015059. https://doi.org/10.1061/(ASCE)CP.19435487.0000540

Frigo M, Johnson SG (2005) The design and implementation of FFTW3. Proc IEEE 93(2):216-231. https://doi.org/10.1109/ JPROC.2004.840301

Grasselli G (2001) Shear Strength of Rock Joints based on quantified Surface Description. PhD thesis, Ecole Polytechnique Federale de Lausanne

Grasselli G, Egger P (2003) Constitutive law for the shear strength of rock joints based on three-dimensional surface parameters. Int J Rock Mech Min Sci 40:25-40. https://doi.org/10.1016/S1365 $-1609(02) 00101-6$

Gravanis E, Pantelidis L (2019) Determining of the joint roughness coefficient (JRC) of rock discontinuities based on the theory of random fields. Geosciences 9(7):295. https://doi.org/10.3390/ geosciences 9070295

Hastings HM, Sugihara G (1994) Fractals-a user's guide for the natural sciences. Oxford University Press, Oxford

ISO (1997) International organization for standardization: geometrical product specifications (GPS) — surface texture: profile methodterms, definitions and surface texture parameters (standard no. 4287)

ISRM (1978) Suggested methods for the quantitative description of discontinuities in rock masses. Int J Rock Mech Min Sci Geomech Abst 15:319-368

Jang H, Kang S, Jang B (2014) Determination of joint roughness coefficients using roughness parameters. Rock Mech Rock Eng 47:2061-2073. https://doi.org/10.1007/s00603-013-0535-z

Kulatilake P, Balasingam P, Park J, Morgan R (2006) Natural rock joint roughness quantification through fractal techniques. Geotech Geol Eng 24:1181-1202. https://doi.org/10.1007/s10706-005-1219-6

Ladanyi B, Archambault G (1970) Simulation of shear behaviour of jointed rock mass. Proceedings 7th Symp. Rock Mechanics, June 16-19, 1969, Berkeley, CA

Lee Y-H, Carr JR, Barr DJ, Haas CJ (1990) The fractal dimension as a measure of the roughness of rock discontinuity profiles. Int J Rock Mech Min Sci Geomech Abst 27:453-464. https://doi. org/10.1016/0148-9062(90)90998-H

Li Y, Huang R (2015) Relationship between joint roughness coefficient and fractal dimension of rock fracture surfaces. Int J Rock Mech Min Sci 75:15-22. https://doi.org/10.1016/j.jirmms.2015.01.007

Li Y, Zhang Y (2015) Quantitative estimation of joint roughness coefficient using statistical parameters. Int J Rock Mech Min Sci 77:27-35. https://doi.org/10.1016/j.ijrmms.2015.03.016

Li Y, Xu Q, Aydin A (2017) Uncertainties in estimating the roughness coefficient of rock fracture surfaces. Bull Eng Geol Environ 76:1153-1165. https://doi.org/10.1007/s10064-016-0994-z

Maerz N, Franklin J, Bennett C (1990) Joint roughness measurement using shadow profilometry. Int J Rock Mech Min Sci Geomech Abst 27:329-343. https://doi.org/10.1016/0148-9062(90)92708-M

Magsipoc E, Zhao Q, Grasselli G (2019) 2D and 3D roughness characterization. Rock Mech Rock Eng. https://doi.org/10.1007/s0060 3-019-01977-4

Malinverno A (1990) A simple method to estimate the fractal dimension of a self-affine series. Geophys Res Lett 17:1953-1956. https ://doi.org/10.1029/GL017i011p01953
Mandelbrot B (1985) Self-affine fractals and fractal dimension. Phys Scr 32:257-260. https://doi.org/10.1088/0031-8949/32/4/001

Marsch K (2020) Rock surface roughness determination: Matlab scripts of evaluation algorithms and original scanned surfaces. https://doi. org/10.14279/depositonce-9592

Marsch K, Wujanz D, Fernandez-Steeger TM (2020) On the usability of different optical measuring techniques for joint roughness evaluation. Bull Eng Geol Environ 79:811-830. https://doi. org/10.1007/s10064-019-01606-y

Myers N (1962) Characterization of surface roughness. Wear 5:182189. https://doi.org/10.1016/0043-1648(62)90002-9

Odling NE (1994) Natural fracture profiles, fractal dimension and joint roughness coefficients. Rock Mech Rock Eng 27:135-153. https ://doi.org/10.1007/BF01020307

Pickering C, Aydin A (2016) Modeling roughness of rock discontinuity surfaces: a signal analysis approach. Rock Mech Rock Eng 49:2959-2965. https://doi.org/10.1007/s00603-015-0870-3

Renard F, Voisin C, Marsan D, Schmittbuhl J (2006) High resolution 3D laser scanner measurements of a strike-slip fault quantify its morphological anisotropy at all scales. Geophys Res Lett 33:L04305. https://doi.org/10.1029/2005GL025038

Schmittbuhl J, Vilotte JP, Roux S (1995) Reliability of self-affine measurements. Phys Rev E 51:131-147. https://doi.org/10.1103/PhysR evE.51.131

Stigsson M, Mas Ivars D (2019) A novel conceptual approach to objectively determine JRC using fractal dimension and asperity distribution of mapped fracture traces. Rock Mech Rock Eng 52:1041-1054. https://doi.org/10.1007/s00603-018-1651-6

Tatone B (2009) Quantitative characterization of natural rock discontinuity roughness in-situ and in the laboratory. Master's thesis, University of Toronto

Tatone B, Grasselli G (2010) A new 2D discontinuity roughness parameter and its correlation with JRC. Int J Rock Mech Min Sci 47:1391-1400. https://doi.org/10.1016/j.ijrmms.2010.06.006

Tse R, Cruden DM (1979) Estimating joint roughness coefficients. Int J Rock Mech Min Sci Geomech Abst 16:303-307. https://doi. org/10.1016/0148-9062(79)90241-9

Turk N, Greig MJ, Dearman WR, Amin FF (1987) Characterization of rock joint surfaces by fractal dimension. Proceedings 28th Symp. Rock Mechanics, June 29-July 1, 1987, Tucson, AZ

Wang L, Wang C, Khoshnevisan S, Ge Y, Sun Z (2017) Determination of two-dimensional joint roughness coefficient using support vector regression and factor analysis. Eng Geol 231:238-251. https:// doi.org/10.1016/j.enggeo.2017.09.010

Wang C, Wang L, Karakus M (2019) A new spectral analysis method for determining the joint roughness coefficient of rock joints. Int J Rock Mech Min Sci 113:72-82. https://doi.org/10.1016/j.ijrmm s.2018.11.009

Yang ZY, Lo SC, Di CC (2001) Reassessing the joint roughness coefficient (JRC) estimation using Z2. Rock Mech Rock Eng 34(3):243251. https://doi.org/10.1007/s006030170012

Yong R, Ye J, Liang Q-F, Huang M, Du S-G (2018) Estimation of the joint roughness coefficient (JRC) of rock joints by vector similarity measures. Bull Eng Geol Environ 77:735-749. https://doi. org/10.1007/s10064-016-0947-6

Yu X, Vayssade B (1991) Joint profiles and their roughness parameters. Int J Rock Mech Min Sci Geomech Abst 28:333-336. https://doi. org/10.1016/0148-9062(91)90598-G

Zhang G, Karakus M, Tang H, Ge Y, Zhang L (2014) A new method estimating the 2D joint roughness coefficient for discontinuity surfaces in rock masses. Int J Rock Mech Min Sci 72:191-198. https://doi.org/10.1016/j.ijrmms.2014.09.009

Publisher's Note Springer Nature remains neutral with regard to jurisdictional claims in published maps and institutional affiliations. 